\title{
Knowledge Sharing Framework: a Game-Theoretic Approach
}

\author{
Rami S. Al-Gharaibeh ${ }^{1}$ (D) Mostafa Z. Ali $^{1}$
}

Received: 26 February 2020 / Accepted: 30 November 2020/ Published online: 30 January 2021

(C) The Author(s), under exclusive licence to Springer Science+Business Media, LLC part of Springer Nature 2021

\begin{abstract}
Uncertainty in business environments is promoting learning as an organizational value. Organizations need to implement knowledge management (KM) processes as well as organizational mechanisms transforming collective knowledge into a learning organizational capability. Literature identifies knowledge sharing (KS) as a fundamental KM process. Moreover, KM was found to be a prerequisite to a learning organization. Unfortunately, organizational initiatives promoting KS are challenged with the hoarding wisdom, "knowledge is power." Literature has researched intrinsic and extrinsic motivations affecting KS intention. The rational action theory (RAT) explains the embedded utility function merging these motivations. Despite many studies, the dynamics of KS behavior needs further examination. This paper is an attempt to frame the KS behavior using game theory and RAT. We represent individuals' perceived utility in two functions: knowledge and trustworthiness. This limits the perceived utility to personal enjoyment and reciprocity, which could be viewed as establishing a baseline KS behavior. We use the assurance game framework to incubate the two utility functions. Finally, we argue that KS intention is actually a dynamic state within a $K S$ strategy. We identify five KS strategies: cooperation, defection, tit-for-tat, unforgiving, and random. It is the performance of these strategies that needs to be studied. Several scenarios are simulated to observe the progression of knowledge within each strategy. Interestingly, two strategies start with positive KS intention yet end up converging with those who started with negative KS intention. On the long run, only cooperatives seem to be contributing to collective knowledge. Empirical data from a teaching hospital is collected and analyzed for comparison.
\end{abstract}

Keywords Game theory · Knowledge management - Learning organization · Modeling and simulation $\cdot$ Knowledge sharing $\cdot$ Rational action theory

Rami S. Al-Gharaibeh

rami@just.edu.jo

Extended author information available on the last page of the article 


\section{Introduction}

Research proposes several definitions for knowledge. One practical definition that relates to organizational performance identifies knowledge as the capability of choosing and implementing a rational action for a certain purpose (Guo and Sheffield 2006). The term experts refer to organizational members with high level of job-related knowledge. The Dreyfus' five-stage model of adult skill acquisition (Dreyfus and Dreyfus 1986) proposes five stages through which an expert develops his expertise: (1) novice, (2) advanced beginner, (3) competence, (4) proficiency, and (5) expertise. The authors attribute the escalation through the stages to a change in the individual's mode of skill acquisition. A mode of analytical learning gradually replaces a mode of procedural or instructional learning. Thus, it is not only the amount of knowledge that makes a person an expert; moreover, it is the way he views and formulates problems (Suzuki and Harnisch 1995).

A business situation could embrace many interdependent factors for which causes and effects are not obviously related. When dealing with such situations, individuals need to assess critically. Knowledge management (KM) emerged as a discipline for formalizing organizational practices that would guide an individual through the skill acquisition journey as effectively and efficiently as possible. The KM research identifies several KM processes including knowledge creation, knowledge acquisition, knowledge sharing, knowledge storage, and knowledge application (Gumus 2007). Some researchers argued that acquisition, sharing, and application are the fundamental knowledge processes (Karkoulian et al. 2013). Others like Nonaka and Takeuchi (1995) developed a model that explains knowledge creation based on four processes: socialization, internalization, combination, and internalization. Socialization could be related to Dreyfus' model of skill escalation through analytical learning since it involves the transfer of tacit knowledge through a social-based process. Thus, socialization is a process demanding knowledge networks to ensure employees get opportunities to share knowledge (McGurk and Baron 2012).

Although KM manages individuals' learning, the organization as an entity needs also to be able to learn (Watkins and Golembiewski 1995). The KM processes that advance individuals' collective knowledge are not appropriate to support organizational learning. According to Senge (1994), organizational learning processes should support five concepts: (1) shared vision, (2) mental models, (3) team learning, (4) personal mastery, and (5) systems thinking. Kim (1993) developed a model linking personal learning with organizational learning. According to the author, the implementation of a learning organization is confronted with a nontechnical obstacle. Organizational politics turns KS into a game. Those who work in teams and decide to share their knowledge perceive their personal knowledge as power, which makes KS a surrender of personal competitive advantage (Cameron 2000; Anand et al. 2020). On the other hand, those who continuously hoard are selfishly accumulating more competitive advantage. Research have showed that the initial KS intention is affected by cultural values. Multinational organizations with diverse cultural backgrounds would have a mixture of sharers and hoarders. As individuals start their engagement in KS activities, they would be applying their initial KS intention. This initial intention would be modified by personal enjoyment as an intrinsic factor and by sense of reciprocity and rewards as extrinsic factors. These effects on the knowledge transmitter do not have 
constant values across the several rounds of KS activities. Meaning, the values are dependent on the evolving behavior of the opponent individual. Thus, while a knowledge sharer would be described as cooperative with a certain opponent, he would in parallel demonstrate defective behavior with another opponent. This reveals that a KS intention is actually a dynamic state within a KS strategy (Rapoport and Chammah 1965).

Rapoport and Chammah (1965) described five strategies for cooperation. Three of them have cooperation as the initial intention, while one strategy starts with a defection intention. The fifth strategy selects its first intention randomly. These strategies describe the cooperation dynamics over a series of cooperation opportunities involving different interacting strategies. The strategies take a game-theoretic perspective towards an individual's intention. The individual is a player in a game where he starts with an initial intention, but as he develops experience, his intention becomes reactive to his opponent's intention. These strategies coincide with the KS research revealing the effect of interpersonal trust among team members. The trustworthiness of the hoarders, by their team members, would decay over time reaching a threshold level below which they would be denied of receiving new knowledge. Accordingly, as a person's trustworthiness among his team members erode, we should expect him to start receiving less knowledge. If every player eventually turns into a defector, the process of KS would stall. This state is against the implementation of organizational learning.

Game theory, being multiplayer decision theory, has an unmined potential as a tool for studying and analyzing organizational cooperation and conflicts of KS. The choice of a player would affect his own utility as well as the utility for opponent players; hence, this would affect decision behavior (Gintis 2009). In the case of a two-player game, we represent the decisions and the utility in a $2 \times 2$ matrix. The matrix produces four quadrants in which the utility for both players is inserted. The most famous example for a one-round game is the Prisoner's Dilemma (PD). The one-round game assumes that a player has no knowledge of his opponent's intention and thus would make his decision solely on the perceived personal utility. The analysis of PD leads to concluding that it has one Nash equilibrium situation that is in the case when both players defect. However, when the two players repeatedly play the game, the PD representation becomes inadequate. This is because each player has now an experience with his opponent's intentions. This latter game has been described as the Assurance Game (AG) (Fehr and Gachter 2000). Interestingly, AG has two equilibria situations, when both players defect or when both players cooperate.

This paper is an attempt to improve the understanding of the dynamics of KS. We start by improving the simple perception of individuals as either knowledge sharers or hoarders. For this purpose, we embrace Rapoport and Chammah's (1965) five cooperation strategies in which continuous sharing and continuous hoarding are two of these strategies along with tit-for-tat, unforgiving, and random strategies. The concept of KS strategy is able to accommodate the current literature as well as paving the way to enrich the understanding of the dynamics of KS. Intrinsic motivations affect an individual's selection of his KS strategy. Included in these factors are the cultural factor and the individual's perceived utility of the KS process, such as enjoyment. On the other hand, the literature has identified extrinsic factors such as reciprocity and organizational rewards. We are proposing a KS framework that revolves around the concept of KS strategy. Both intrinsic and extrinsic motivations either affect the 
selection of the strategy or provide feedback for strategy implementation. A good start in the application of this approach would be to establish a baseline KS behavior, that is, a behavior with no organizational intervention. We are establishing this baseline behavior by developing a KS framework with an individual's utility perception that is free from organizational rewards.

Regardless of the KS strategy, an individual uses his own perceived utility function to decide for each KS opportunity whether to share or hoard. Rational action theory (RAT) explains the KS decision by theorizing that an individual would make a sharing decision when benefits of sharing exceed its costs. In our proposed KS framework, we recognize the following elements to be the traded benefits and costs: (1) knowledge, (2) competitive advantage, and (3) trustworthiness. Again, organizational rewards are not considered in order to achieve the purpose of examining the dynamics of interventionfree KS. We develop two utility functions: (1) knowledge utility function and (2) trustworthiness utility function. The former represents the exchange of knowledge and competitive advantage, while the latter represents the exchange of trustworthiness. The last step is to insert this setup within a game framework. This is important to capture how the utility function is used with different opponent strategies. In every round of the game, the player facing a KS decision will assess his utility in this situation with a specific opponent. According to Wolfe and Loraas (2008), regardless of incentive type, the incentive utility must be perceived sufficient in order to promote a sharing decision.

Although PD is the most famous game, it cannot provide a suitable framework because it does not support the concept of reciprocity. PD assumes that the players play only one round, and thus the rational action would lead both players to defect and reach the one equilibrium situation. However, the AG is memory based, as it assumes that the same players will meet over several rounds, and thus the concept of reciprocity is implemented. A player will start with a positive KS intention and will maintain it for as long as he trusts that the opponent has a sufficient level of trustworthiness. Our KS framework implements reciprocity through three mechanisms: (1) individuals with titfor-tat KS strategy, (2) individuals with unforgiving KS strategy, and (3) individuals with cooperative KS strategy and sufficient trustworthiness level.

Finally, we setup parameters for the utility functions and prepare our framework for simulation. The result that we are monitoring is the progress of a strategy's percentage contribution to collective knowledge, which is the raw material for organizational learning. For a baseline scenario, it is fair to assign equal number of individuals for each strategy, 20 players. This scenario implies that about 70 individuals will start the game with a sharing intention and 30 players with a hording intention. In this scenario, the defective strategy performed the poorest. In the second scenario, we increased the number of defectors to 50 while maintaining 20 individuals in the other strategies. This equalizes the number of initial sharers and hoarders. This formation enhanced the performance of the defectors against the random strategists, but not against other types of strategists. Moreover, the knowledge progression graphs revealed that competition laid between the two strategies of cooperative and tit-for-tat, with long-run supremacy for cooperative. Hence, we attempted a third scenario with number of tit-for-tat players increasing to 50 and for other strategies maintained at 20 players. This gave more supremacy for tit-fir-tats on the short run, yet cooperative players eventually took over the lead. All other scenarios would lead to the same long-run conclusion but with different short-run relative performances. 
The simulations with our proposed KS framework reveals that when a group of individuals with different KS strategies are engaged in a series of KS activities, they will eventually settle in two equilibria states, as the assurance game suggests. All players with cooperative strategy continue learning at an increasing rate and would thus be contributing the highest percentage of collective knowledge. All other strategies, regardless of their initial KS intention, will converge towards a poor state of learning with poor contribution to collective knowledge. This conclusion challenges the common hoarding wisdom that "knowledge is power." On the contrary, it shows that "knowledge sharing is power." It seems that the wisdom depends on which window in time you examine the KS behavior. Our simulation shows that many rounds of KS activities are required for the advantages of KS to payoff. It is this big time lag that is discouraging the adoption of the cooperative strategy. Future expansion on this research would add organizational rewards to the perceived utilities and test the added motivation on the baseline behavior that we have established.

As a preliminary preparation for a longitudinal verification study, we attempted a survey-based empirical study. The results verified the divergence of KS intentions among residential physicians in a teaching hospital along two trends towards two equilibrium situations as the AG suggests. Also, individuals with initial sharing intentions expressed a decrease in their trust towards their colleagues, with an increase in their sensitivity against repeated defections. Decrease in trustworthiness was .053 in correlation with decreases in KS intentions among junior residents, yet they were .45 in correlation among senior residents.

\section{Literature Review}

There is agreement among researchers that turbulence in business environments has been rapidly increasing. Organizational survival has become very dependent on the organization's ability to monitor and adapt. Senge (1994) cites a study carried out by Royal Dutch/Shell in 1983 revealing that third of the companies in the Fortune 500 in 1970 has disappeared. He relates this phenomenon to the way firms are managed and the ways employees think and interact. As this realization became common, attention to learning as core business value came under attention. In fact, learning could be the only sustainable organizational competitive advantage (Senge 1994; Karkoulian et al. 2013).

The term learning organization (LO) came to existence to describe a firm that encourages employees to acquire new knowledge for the aim of solving problems in any area of the organization (Goh 2003). Kline and Saunders (1993) described a 10step process for building an LO. The process addresses personal issues such as attitude and behavior, knowledge sharing, and team problem-solving, as well as organizational issues such as setting up a learning culture and a rewarding system. The authors highlight the need to harness the collective learning at the personal level to produce organizational learning and benefits.

Goh (2003) carried out extensive review of literature on LO and identified five characteristics of an LO: (1) clarity of mission and vision, (2) leadership commitment and empowerment, (3) experimentation and rewards, (4) effective transfer of knowledge, and (5) teamwork and group problem-solving. The author designed a study to test the progress of learning capability of an organization applying these five building 
blocks. In his first case study, he applied his longitudinal survey to a high-technology research institution; such institution is exemplary for its learning orientation. The results showed improvement in learning along three dimensions with poor improvement in the two dimensions of experimentation and reward and teamwork and group problem solving. His second case study was conducted on a firm that develops new products and marketing strategies for telephone companies. The results for this case showed improvement in learning capabilities along all five dimensions.

The outcome of learning, whether at a personal level or organizational level, is knowledge. The knowledge that personal learning produces is the capability of choosing and implementing a rational action for a certain purpose (Guo and Sheffield 2006). Since the business environment is changing, the rational actions by individuals are not expected to be constant. Although organizational management of its knowledge is as old as organizations existed, the discipline of KM emerged to formalize its organizational practices (Karkoulian et al. 2013). Swan et al. (1999) identified KM as the organizational practice of capturing, creating, acquiring, sharing, and applying knowledge. In a similar fashion, Gumus (2007) defines KM as the collection of processes that support creation, storage, sharing, and evaluation of knowledge. Eventually, these KM processes are to be integrated with work processes to lead to the ultimate goal of correcting dysfunctional organizational behavior relative to emerging environmental conditions (Labedz et al. 2011).

With LO and KM both researching learning within organizations, a need for understanding the relationship became essential. One way to envision this relationship is to think that as individuals acquire personal knowledge, a corporate structure is required to leverage collective personal learning to an organizational level. According to Aggestam (2006), KM is the starting practice that paves the way to LO, yet there is a feedback from LO that affects the triggering KM. To assess the interdependence between LO and KM, Karkoulian et al. (2013) conducted an empirical research with the assumption that KM is represented in three processes: knowledge acquisition (KA), knowledge sharing (KS), and knowledge utilization (KU). On the other hand, LO is represented in mechanisms such as continuous learning, empowerment, and team learning. Their results confirmed Aggestam's (2006) theorizations that KM processes do trigger the successful development of LO more than LO processes enhance KM. Similarly, Dahou et al. (2019) conducted a case study for the purpose of researching the effect of KM on the development of OL capability (OLC). The researchers in this study represented KM using the four conversion processes of the SECI model (Nonaka and Takeuchi 1995). In consistence, the results supported the envision of KM being the trigger of LO, with socialization process (comparable to KS) being the major influencing factor.

According to Alavi and Leidner (2001), knowledge is the result of cognitive process triggered by new information. The resulting knowledge could then be retransformed back to information by a process of articulation (Acar and al-Gharaibeh 2019). This envision leads to the conclusion that learning at an organizational level is to build organizational and collective understanding of the newly articulated information. This objective requires an organizational culture of KS intention. Actually, one observation that Karkoulian et al. (2013) revealed in their study is that a KS organizational culture is important to enhance KU, which is the ultimate goal of learning. Thus, for the achievement of the sustainable competitive advantage and the value of learning, a 
KS intention is fundamental. McGurk and Baron (2012) highlight the importance of increasing the opportunities for KS through mechanisms such as knowledge networks. Hoarders of knowledge are of little organizational value. Unfortunately, individuals tend to hoard their knowledge rather than to share it (Bock et al. 2005).

Davenport and Prusak (1998) described how organizational knowledge, in contrast with personal knowledge, is dynamic and is affected by several forces. To be able to utilize organizational knowledge, we have to sufficiently understand KS. According to the authors, organizational KS has a similar dynamic as the market for a tangible good. The knowledge market has transmitters and receivers who negotiate to reach a satisfactory price for the exchanged knowledge. Individuals seek knowledgeable people in order to acquire solutions to problems. On the other side, knowledgeable people expect to benefit from their supply of knowledge. Although KS transactions do not involve cash, a system of price and payment do exist. The parties involved in KS are exchanging scarce units for present or future values. A utopian managerial vision that knowledge is shared without friction is unrealistic. Individuals do not normally give away personal possessions, including knowledge, without expecting a benefit in return.

Many studies attempted to investigate the KS intention and the factors that affect it. According to Hau et al. (2012), these studies reveal intrinsic and extrinsic types. Intrinsic motivation refers to the motivation to perform an activity for nonmonetary perceived utility such as the enjoyment of performing the activity per se. Empirical research has shown a positive correlation between enjoyment and knowledge sharing intention (Wasko and Faraj 2000). Intrinsic motivation by itself is affected by cultural values. For example, Hsu (2006) conducted a study on nine manufacturing firms in Taiwan. The data analysis revealed that the cultural values of Taiwan promotes knowledge hoarding. The effect of cultural values was also investigated by Sandhu and Ching (2014) in a study that targeted this effect in multinational corporations (MNC) operating in different countries. In such diverse cultural environments, KS could become very challenging. The researchers collected data from 231 senior officers in selected MNCs in Malaysia. The results confirmed that initial KS intentions are different across individuals from a variety of cultural values and backgrounds. According to the researchers, this effect could be reduced by identification of individuals with undesirable initial KS intentions at the recruitment stage. Or, by setting up organizational practices (such as rewards) that would correct the initial undesirable KS intentions. Similarly, Zhang et al. (2014) investigated effect of national cultural values on KS within a multinational virtual class. The authors conducted a qualitative case study with 6 semi-structure interviews and a cross-sectional survey to examine the interaction effects of culture and their different KS intention. Interestingly, the authors found that some aspects of the cultural values affected the initial KS intentions, while others demonstrated interactive effect with KS behavior.

On the other hand, extrinsic motivations include monetary utility such as organizational rewards, as well as nonmonetary utility such as the sense of reciprocity. However, there have been contradicting conclusions about the effect of extrinsic motivations ranging from positive correlation to no correlation and even to negative correlation (Kankanhalli et al. 2005; Hung et al. 2011; Bock et al. 2005). Still, many organizations revert to rewards as a mechanism for improving KS intentions. The conception is based on the rational action theory. Since hoarding is motivated by a sense of benefit, then a reward with a higher value should be offered to compensate the 
perceived benefits of hoarding (Mueller et al. 2005). For example, Lam and Lambermont-Ford (2010) suggested that KS may be encouraged through normative motivation in combination with pleasant motivation. Hung et al. (2011) studied the effects of both intrinsic and extrinsic motivations on $\mathrm{KS}$ and found that reputation feedback supports successful KS in a knowledge management system (KMS). The effect of reputation has been previously discussed by Davenport and Prusak (1998) as a factor supporting reciprocity. Reciprocity explains the knowledge sharer's initial intention to share his knowledge because he expects to be treated equally in future knowledge sharing opportunities. The initial sharing intentions would be reversed when reciprocity proves disappointment.

Reciprocity is dependent on the level of trustworthiness as a feedback (Davenport and Prusak 1998). Bligh (2017) defines trust as a person's level of reliance on another person's actions, words, and good intentions in fulfilling his promises. The observational study by Lee et al. (2010) found positive and significant relationship between trust within the team and the level of internal KS. Another study by Chen and Hung (2010) also identified interpersonal trust and perceived relative advantage as drivers of KS behavior. Likewise, Politis (2003: p64) concludes: "Such a 'trustworthy' intention among co-workers is the chief ingredient for knowledge acquisition and knowledge sharing." A recent empirical study by Ogunmokun et al. (2020) collected data from 180 restaurants. A sample of 453 employees was used to test the research hypotheses. The results showed that propensity to trust is positively related to KS behavior. Another factor that might increase the cost of KS is the organizational KS facilitation. According to Cabrera and Cabrera (2002), a perception of high cost a KS process exerts on a knowledge sharer could reverse his initial intention from sharing to hoarding. The organizations should be keen on designing the $\mathrm{KS}$ process in a way making it convenient to share knowledge. This was also suggested by Zhang (2018) after modeling incentives and information technology as the facilitator of knowledge management processes. So, the contradicting results about the effect of extrinsic motivations might have to do with inappropriate framing of the KS dilemma. An appropriate framing would allow for two things: (1) a differentiation between the initial intention, and the postreciprocity intention, and (2) treating $\mathrm{KS}$ on opponent basis rather than flat and generalized basis. For this purpose, an individual's KS intention should be embedded as a dynamic state within a KS strategy that has an initial intention value. The KS strategy describes the mechanism guiding a series of future decisions rather than describing the status of one single KS decision. The validity of the revised literature is still recognized as factors affecting a KS strategy rather than the dichotomous KS intention.

Rapoport and Chammah (1965) suggested a decision strategy for a game player which they called tit-for-tat. The game is a two-player game going through a series of cooperation opportunities where in every opportunity each player would have to decide whether to cooperate or defect. A player adopting this strategy would start the first round with a sharing intention. In the following rounds, the player decides whatever his opponent has decided in the previous round. The authors also described two extreme strategies where the player is unaffected by his opponent's decision: the cooperation strategy and the defection strategy. A 
cooperative player is an altruist who is happy to continuously share regardless of his opponent's decisions. On the other extreme, a defective player is one who constantly demonstrates hoarding intention. Some players would start as sharers and maintains this behavior until they are met with an opponent's hoarding decision. Only one hoarding incident is enough for such players to switch from being cooperative to being defective; this is referred to as the unforgiving strategy. Lastly, the authors described a player with a random behavior where his intention in each round is independent of any factor.

Game theory is not viewed as an abstract mathematics but rather as a fundamental concept that is concerned with cognitive capabilities and conflicts among players as in real-world scenarios (Rubinstein 1991). Researchers view game theory as a nonconcrete inquest into the logic and function of patterns of behavior and social interactions. The applicability has grown from simple analysis of abstract cooperation/conflict in economics to biology (Maynard-Smith 1982) and to a whole spectrum of behavioral sciences (Gintis 2009). In decisionmaking scenarios involving multiple individuals, game theory is considered very useful for analyzing strategic interdependence using backward reasoning (Myerson 1997; Chuanrong and Deming 2009) and understanding counterintuitive insights. Equilibrium analysis deals with analyzing and discovering equilibria situations (Camerer 1991). In this type of analysis, game-theoretic reasoning, among other types of equilibrating forces, tries to reach equilibrium (Dixon 2000). When using games to study business environments to provide practical reasoning using models, equilibration in games could be reached using other forces like communication, evolution, and introspection (Lynne 2001; Wu and Xuan 2004).

Mueller et al. (2005) and Zhang et al. (2010) both studied the combined effect of incentives and culture on KS. For that purpose, they viewed KS as a social dilemma and theoretically studied the dynamics of KS using the Prisoner's Dilemma (PD) framework with two players facing two types of decisions (sharing/hoarding). The authors' theoretical argument leads to a Nash equilibrium when both players decide to hoard. The authors simulated the game under controlled lab conditions. Strangely, their experimentation results showed a higher cooperation rate than the PD model predicted. We could explain this contradiction in the missing representation of the reciprocity concept. In the case of KS where the same players repetitively engage in the game, the PD model is not representative. The PD should be replaced with the Assurance Game (AG) model (Fehr and Gachter 2000). An AG game has two Nash equilibria situations, as opposed to the one situation in a PD game. The AG framework implements reciprocity which could be described as conditional cooperation. A player is willing to cooperate if his opponent cooperates as well and will stop the cooperation if the opponent stops cooperating. Accordingly, each player will memorize the decision of each opponent in the previous interaction. In our framework, we are modeling this player's memory with a trustworthiness utility function. The integration of the KS strategies with the AG does not contradict the conclusions from previous literature. For example, the established conclusions about the effect of cultural values on KS intentions could be explained as these cultures are generating more players with a defective strategy. 


\section{Preliminaries}

\section{Game Theory}

Game theory is mainly defined as the study and analysis of mathematical models of conflict and cooperation in a proposed environment with some constraints (Myerson 1997). Game theory is divided into cooperative and non-cooperative game theory. Cooperative game theory is an effective concept in the context of coalitions like in political science. On the other hand, non-cooperative game theory is useful in analyzing situations in static and dynamic games where available information is incomplete like in playing chess or poker.

In game theoretical terms, a game $\mathfrak{I}$ is a social context created among a set of players $(N P)$. The players are assumed to be rational with a certain level of intelligence. In organizational context, a decision-maker is considered to be rational when the outcomes of his decisions are congruent with the organizational objectives. In the game $\mathfrak{I}$, each player follows a certain strategy $S_{\mathrm{i}}$ that guides the decisions of the player and thus determines the utility. Despite the different strategies, the goal remains one: to maximize an individual's utility. This paper investigates the changes in the player's knowledge $\mathrm{kg}$ as part of the utility resulting from the player's strategy. Assuming that the resulting knowledge volume for individual $i$ following a knowledge sharing opportunity is denoted as $k g_{v}^{i}$, then the basic model of $\mathfrak{I}$ is represented as:

$$
\mathfrak{I}=\left(N P,\left(S_{i}\right)_{i \in N P},\left(k g_{v}^{i}\right)_{i \in N P}\right)
$$

\section{The Prisoner's Dilemma}

Prisoner's Dilemma (PD) is the first thing that comes to mind when game theory is mentioned. The PD was introduced by Von Neumann and Morgenstern (1944) with over a thousand related publications before the 1970s (Donninger 1986). The PD is the dilemma between selfishness and altruism. In a scenario where there is only one player, the best strategy for a rational selfish player is to defect. In this case, defection is the dominant strategy, and it makes no difference about the rationality of the other player. Different versions of the PD were introduced demonstrating different applications (Bhaskar and Obara 2002; Mailath and Morris 2006; Piccione 2002). These versions were studied from different perspectives, mainly repetition and monitoring. In most of these previous works, the concept was introduced to inspect the rationale behind the PD for international business using a multinational enterprises (MNE) perspective.

\section{The Assurance Game}

Game theory offers a proper scheme for the analysis of social dilemmas. The most common and investigated cases involved two-player interactions and three fundamental types of social dilemmas: the Chicken, the PD, and the Stag Hunt games (assurance games) (Kramer et al. 1995; Skyrms 2003). Different kinds of social tension portray and describe such dilemmas and are referred to as greed and fear, with two motives lie 
behind non-cooperative behavior of players. The greed motive describes situations in which players favor unilateral defection to mutual cooperation. The fear motive describes cases in which players favor mutual defection over unilateral cooperation. Only fear is present in stag hunt games.

To study the role of trust in KS and the resulting collective knowledge, "assurance game" or "stag hunt" model situation shall be used. Based on a story of Rousseau, "stag hunt" is described for cases where two people must choose between hunting hare and stag. The process of hunting stag pays off well if successful; however, the process fails if the person attempts it with no help. The process of hunting hare can be done successfully when the person attempts it alone and the payoff is considered moderate. In the stag hunt model, the process encompasses many situations where the measure of how well the group does is based on how the members of the group coordinate on a cooperative endeavor but where this attempt fails if a defection is detected (Kramer et al. 1995; Skyrms 2003; Cooper 1999; Kollock 1998). The payoff rules are shown in Table 1. It describes all combinations of the choices of two players between the risk-free approach to defect and the risky approach to cooperate.

From a game-theoretic analysis perspective, the assurance game has two stable Nash equilibria: the first equilibrium is the risk-dominant one where both players defect (obtaining a modest payoff), and the second equilibrium is the efficient one where both players cooperate (obtaining a high payoff). The "Prisoner's Dilemma" is a related game that is frequently used in studying cooperation. In PD, an equilibrium is achieved only through mutual defection. The Prisoner's Dilemma can be transformed into a stag hunt via repeated interactions, according to the folk theorem (Sen 1967). People tend to think of Prisoner's Dilemmas and perceive it as stag hunts (Kiyonari et al. 2000), and stag hunt has been shown in theoretical work to be of more importance for ingroup preferences to evolve (Jansson 2015). The problem of determining whether to dedicate energy to establish a fresh mutually positive social pact or not is a real-life example of an assurance game with many applications. The existence of two-way communication makes cooperation in the stag hunt highly likely (Clark et al. 2001; Cooper et al. 1994). Communication is a way to rise a common group identity. The latter is an important factor to indicate both further cooperative behavior and higher trust (Dawes et al. 1988; Brewer 1979). In fact, the authors in Ahmed (2007) found that the players following a stag hunt strategy cooperated more often when they were divided into minimal groups and when they knew they were paired with a member of the same group.

Table 1 Payoff matrix of the assurance game (stag hunt)

\begin{tabular}{llll}
\hline & Player 2 & \\
\cline { 3 - 3 } & & Cooperation & Defection \\
\hline Player 1 & Cooperation & High [150] & Medium [100] \\
& Defection & Medium [100] & Low [0] \\
\hline
\end{tabular}

Numbers within brackets are the payoffs used in component experiments 


\section{Methodology}

\section{The Knowledge Sharing Framework}

The proposed KS framework is shown in Fig. 1. The framework shows the two types of motivations that have been reviewed to be affecting KS intention. As per our argument, we perceive KS intention to be a dynamic state within a KS strategy. Thus, the intrinsic factors are shown to affect the player's selection of a KS strategy. However, the selection is modified by the player's perception of utility of the KS experience according to the rational action theory. Within the extrinsic motivation, we have highlighted the two types of interpersonal trust and organizational rewards. The organizational rewards when implemented would create a utility matrix that would be perceived by the players and would modify their KS strategy. This is the effect that has been studied in the literature for enhancing tendency towards sharing intention. In our proposed framework, it would be enhancing the tendency towards the adoption of cooperative strategy. It should be remembered that we have not developed the rewards utility matrix because we seek to conclude a KS baseline that is intervention free.

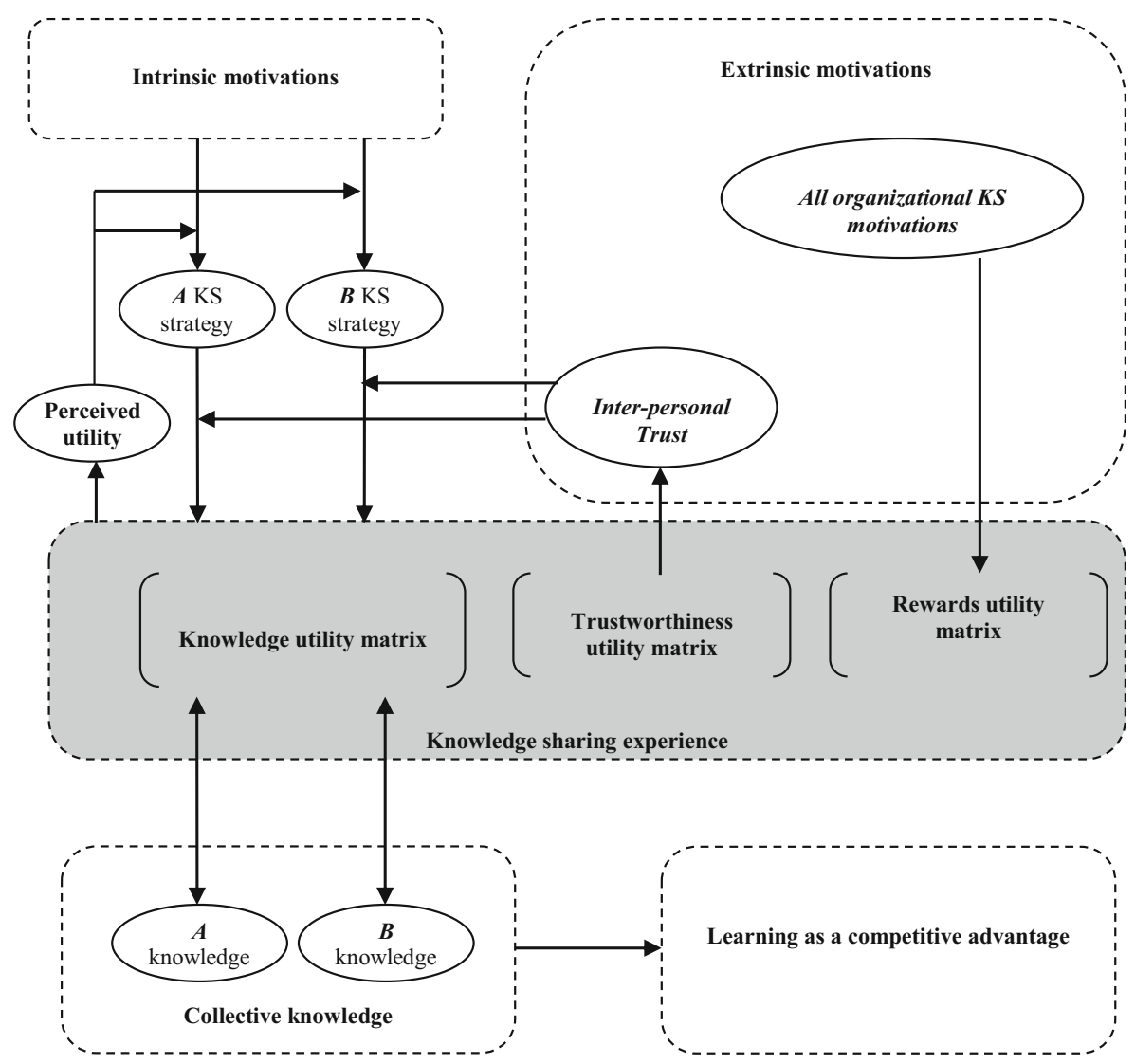

Fig. 1 The proposed knowledge sharing framework 
In the first round of a KS series, two players with randomly selected KS strategy will meet where each of them will make a sharing or hording decision, as his strategy dictates for the first move. The two decisions will trigger both matrices within the KS experience and will produce for each player three changes: (1) a change in knowledge, (2) a change in competitiveness, and (3) a change in trustworthiness. The framework shows that the change in trustworthiness goes back as input to feed a second round of KS with this opponent. The newly created knowledge within each player is considered part of the organizational collective knowledge that should fuel organizational learning. This is actually the outcome that we are concerned with observing its progression. Precisely, we are observing the contribution of each type of strategy in feeding this outcome.

By modeling this framework, we could form different combinations of KS strategies, thus simulating employees engaged in KS activities. The simulations will enable us to observe the value of each strategy in its contribution to organizational learning. In the next section, we describe the development of the two utility matrices.

\section{Knowledge Utility Matrix}

Organizational promotion of KS could be perceived as a game between the organization itself and its employees. We assume $\mathrm{kg}_{v}^{i}$ to be an employee's volume of knowledge. When an employee transfers his knowledge to a colleague, he bears costs denoted as $C_{\mathrm{KT}}$. The employee is less likely to make a knowledge transfer unless he receives incentives $C_{\text {inc }}$, which is actually cost incurred by the organization. In return for its investment in incentive costs, the firm grows in its organizational knowledge denoted as $f\left(k g_{v}^{i}\right)$. Accordingly, we could develop the utility matrix for knowledge sharing between employees on one hand and the organization on the other hand (Table 2). The matrix reveals that employees perceive KS as risky even when they receive incentives. On each KS opportunity, an employee needs to value his $C_{\mathrm{KT}}$ and make sure that $C_{\mathrm{inc}}$ is higher; otherwise, he will defect the sharing process and still receives the $C_{\text {inc }}$. When the organization realizes that its incentive program is ineffective, it would bring such program to a halt. In return, receiving no compensations for their $C_{\mathrm{KT}}$ would promote defective sharing strategies, and the system would eventually go to equilibrium in the lower right quadrant.

As an introductory investigation, we need to improve our understanding of the employees' baseline KS behavior, that is, the behavior with no organizational interference. In order to develop a KS utility matrix, this research uses the simplest case of two-player game. Knowledge is commonly divided into explicit knowledge and tacit

Table 2 Utility matrix for a game of organizational KS incentives

\begin{tabular}{lll}
\hline Organizational incentives & Employee & \\
\cline { 2 - 3 } & Adopt KT & Reject KT \\
\hline Yes & $\left(f\left(\mathrm{~kg}_{v}\right)-C_{i n c}, C_{i n c}-C_{K T}\right)$ & $\left(-C_{i n c}, C_{i n c}\right)$ \\
No & $\left(f\left(\mathrm{~kg}_{v}\right),-C_{K T}\right)$ & $(0,0)$ \\
\hline
\end{tabular}


knowledge (Nonaka and Takeuchi 1995). When players $A$ and $B$ are engaged in a KS opportunity, each should attempt to transmit his transferrable knowledge. These are denoted as $\mathrm{kg}_{T}^{A}$ (for player $A$ ) and as $\mathrm{kg}_{T}^{B}$ (for player $B$ ). However, more important than acknowledging the transmitted knowledge is identifying the absorbed knowledge at the recipient side. According to Cohen and Levinthal (1990), an individual's ability to absorb and utilize received knowledge is dependent on his related prior knowledge. Thus, the acquired knowledge is not necessarily equal to the transmitted knowledge; it could be a subset of the transmitted knowledge. Moreover, knowledge assimilation and utilization among recipients vary based on their personal absorptive capacities. Our proposed framework represents absorptive capacity denoting it as $\sigma_{A}$ and $\sigma_{B}$ for players $A$ and $B$, respectively.

For the transmission of tacit knowledge, the framework adopts the perspective that it is ineffable. Accordingly, the proliferation of tacit knowledge requires continuous process of knowledge development, which makes it a constructive, interactive, and collaborative process (Tsoukas 1996; D'Eredita and Barreto 2006). The received knowledge triggers inquisitions at the recipient side met by answers at the transmitter side. These inquisitions are the attempt of the recipient to identify where in his structure of prior knowledge would this received knowledge properly and cohesively fit. Several cycles of inquisitions and returned answers would end by a successful fusion of absorbed knowledge with prior knowledge. The level of knowledge acquisition depends on the ability of the recipient to ask the right questions and absorb the returned answers. The interactivity and continuity of this process involve trust between the two players. The validity of this argument has been established, and thus trust has been included as a factor in the framework. A mistrusted player will be deprived of the cooperation of the knowledge sharer and thus of the chance to issue inquisitions and receive answers. The framework refers to this process as knowledge fusion and denote it as $k g_{\text {fus }}^{A}$ and $k g_{\text {fus }}^{B}$ for both players $A$ and $B$, respectively.

With high level of knowledge comes a prestigious status or reputation within a field of expertise. The uniqueness of the status empowers the expert with high competency. With each knowledge-sharing opportunity comes a change in the individual's level of competitiveness. The notation $\mathrm{kg}_{A \leftarrow B}^{A}$ denotes the positive change in the level of competitiveness for player $A$ after receiving knowledge from player $B$. The negative competitiveness that $A$ loses after transferring knowledge to $B$ is denoted as $k g_{c p t v}^{A}$. Having the fundamental variables identified and denoted, the suggested utility function for player $A$ following a KS round would be:

$$
\Delta k g^{A}=\sigma_{A} k g_{T}^{B}+k g_{\text {fus }}^{A}+k g_{A \leftarrow B}^{A}-k g_{c p t v}^{A}
$$

The value of each of the four terms depends on the decision made by player $A$ and his opponent player $B$. For example, in the case of $A$ deciding to share and $B$ deciding to hoard, the utility functions for players $A$ and $B$ would become as follows:

$$
\Delta k g^{A}=-k g_{c p t v}^{A}
$$




$$
\Delta \mathrm{kg}^{B}=\sigma_{B} k g_{T}^{A}+k g_{B \leftarrow A}^{B}
$$

The utility function for each player can now be used to construct a knowledge utility matrix representing the several cases in a gaming environment. Although there are several KS strategies that could be adopted by each player, the decision alternatives remain dichotomous, either share or hoard. The matrix in Table 3 shows the four possible quadrants with each quadrant containing the utility function for each player.

If this game is played as a PD game, then it would have one equilibrium situation, the lower right. However, if it is played as an AG, then it would have two equilibria situations: upper left and lower right. A player will end up in one of the two equilibria situations based on his KS strategy. It is for the advantage of the organization that its employees end up in the upper left equilibrium situation.

In a previous section, we have identified five KS strategies. Three of the strategies are independent of the player's experience with an opponent; these are the cooperative, defective, and random. On the other hand, the two strategies of tit-for-tat and unforgiving are experience dependent. A tit-for-tat player will remember the last decision made by an opponent in the last KS experience and respond similarly. The unforgiving player will always cooperate until only once faced with defection and then will permanently defect with the defector. It is of interest for any organization to discover the knowledge progression curve for each strategy and at which equilibrium situation the strategy would eventually settle.

\section{Trustworthiness Utility Matrix}

Table 3 showed the knowledge utility matrix for a KS experience. However, the decisions to be made on that matrix are a function of a feedback from another matrix that memorizes the opponents' decisions in the previous KS experience. This other matrix is the trustworthiness utility matrix. There are two perceptions of an individual's trust, the first relates to his trust to share knowledge, and the second relates to his trust in seeking knowledge (Hashim and Tan 2015; Zhang and Chen 2018). Research provides evidence that both perceptions jointly come to play at any KS opportunity. Our framework assumes that each player maintains a value of trustworthiness of each opponent $(T)$. This $T$ values an opponent player as a knowledge sharer. The more knowledge he shares, the higher trustworthiness he gains and vice versa. When a couple of players with sharing intentions meet, the player higher in knowledge level

Table 3 The knowledge utility matrix for two-player game

\begin{tabular}{|c|c|c|c|}
\hline & & \multicolumn{2}{|l|}{ Player B } \\
\hline & & Share & Hoard \\
\hline \multirow[t]{2}{*}{$\begin{array}{l}\text { Player } \\
\text { A }\end{array}$} & Share & $\begin{array}{l}\left(\sigma_{A} k g_{T}^{B}+k g_{f u s}^{A}+k g_{A \leftarrow B}^{A}-k g_{c p t v}^{A}, \quad \sigma_{B} k g_{T}^{A}+k g_{f u s}^{B}+k g_{B \leftarrow A^{-}}^{B}-\right. \\
\left.\quad k g_{c p t v}^{B}\right)\end{array}$ & $\left(-k g_{c p t v}^{A}, \sigma_{B} k g_{T}^{A}+k g_{B \leftarrow A}^{B}\right)$ \\
\hline & Hoard & $\left(\sigma_{A} k g_{T}^{B}+k g_{A \leftarrow B}^{A},-k g_{c p t v}^{B}\right)$ & $(0,0)$ \\
\hline
\end{tabular}


will have more to lose in his competitiveness, yet he will compensate that with higher gain in trustworthiness. On the other hand, the sharer who receives more knowledge will lose a proportional fraction of his trustworthiness. Players with hoarding intention will receive double punishment. Firstly, they will lose a fraction of their trustworthiness that is proportional to their gain in knowledge. Secondly, they are punished for their selfish decision by deducting a constant amount of their trustworthiness. A meeting between two players with hoarding intentions involves zero exchange of knowledge; thus, each player is penalized by deducting the amount from his trustworthiness value. Table 4 shows the trustworthiness utility matrix for a two-player game.

A cooperative player will maintain a sharing behavior as long as the opponent's trustworthiness is at least equal to a certain threshold $T_{\text {th }}$. When facing a sharing decision with an opponent whose trustworthiness has decayed below $T_{\text {th }}$, the cooperative player would hoard. This assumption contradicts the theoretical description of an altruist who continuously cooperates regardless of the opponent's trustworthiness. Yet, it is a representation of the research findings that trustworthiness affects KS behavior. On one extreme of this conception lies the unforgiving strategy where the $T_{\text {th }}$ is reached after only one incident of defection. On the other extreme lies the altruist who would never defect. Davenport and Prusak (1998) discuss the concept of reputation as a cooperator. According to the authors, individuals mostly like to advertise themselves as knowledgeable people with willingness to share their knowledge. A good reputation increases the chances of reciprocity, that is, the sharer is more likely to receive cooperation when he is in need for it. In a similar reasoning, a bad reputation should decrease an individual's chances in receiving knowledge. Our introduction of the $T_{\text {th }}$ is the implementation of Davenport and Prusak (1998) concept of repute.

For the two types of tit-for-tat and unforgiving strategies, they maintain their usage of the trustworthiness utility matrix, yet with their character having dominance. Both players will defect with an opponent who defected in their last mutual KS experience, even if the opponent's trustworthiness has not yet expired. A tit-for-tat player will return to using $T_{\text {th }}$ following an opponent's redemption. Since the unforgiving player does not recognize redemption, he would continue his hoarding behavior with the specific opponent. In this case, the utility matrix will start punishing the unforgiving player for his hoarding behavior. A random player is insensitive to using the trustworthiness matrix since he makes random decisions. Still, the opponent players would be using the matrix against him. Finally, a defective player will use the matrix with his opponents, yet he will always perceive that his gains are higher in value than the trustworthiness he loses.

Table 4 The trustworthiness utility matrix for two-player game

\begin{tabular}{|c|c|c|c|}
\hline & & \multicolumn{2}{|l|}{ Player B } \\
\hline & & Share & Hoard \\
\hline \multirow[t]{2}{*}{ Player A } & Share & $\left(\frac{\Delta k g^{B}}{\Delta k g^{A}} T_{0}^{A}, \frac{\Delta k g^{A}}{\Delta k g^{B}} T_{0}^{B}\right)$ & $\left(\left|\frac{\Delta k g^{B}}{\Delta k g^{A}}\right| T_{0}^{A},\left|\frac{\Delta k g^{A}}{\Delta k g^{B}}\right| T_{0}^{B}-\lambda\right)$ \\
\hline & Hoard & $\left(\left|\frac{\Delta k g^{B}}{\Delta k g^{A}}\right| T_{0}^{A}-\lambda,\left|\frac{\Delta k g^{A}}{\Delta k g^{B}}\right| T_{0}^{B}\right)$ & $\left(T_{0}^{A}-\lambda, T_{0}^{B}-\lambda\right)$ \\
\hline
\end{tabular}




\section{Experimenting with the Knowledge Sharing Framework}

\section{Experimental Configuration}

We have made an argument for the introduction of the KS strategies. Based on the developed utility matrices, every strategy would show different performance against each opponent strategy throughout the course of the game. For example, greedy strategies, such as "defective," always perform well against the random strategy but poorly against itself. Tit-for-tat strategy performs against itself in the same fashion as if two cooperative players are playing. However, it performs poorly against the random strategy. Our proposed KS framework allows us to cross-compare the performance of these strategies with different combinations. Most importantly, we are interested in observing this comparative performance over a long period of time. Such an observation has not received enough attention.

The experimental landscape ("world" of the model) is a square region of $130 \times 130$ cells hosting 100 players. Colors are used to indicate the affiliation of a player with a sharing strategy as shown in Fig. 2. The simulator allows the players to randomly move in this confined space. The collision of two players at a surface coordinate represents an organizational problem requiring mutual collaboration between the two colliding players. Despite their variance in KS strategies, all players are attempting to increase their own utility. One key difference among the KS strategies is their reliance on reciprocity. The three strategies of cooperative, tit-for-tat, and unforgiving are all reciprocity dependent. However, they demonstrate different levels of sensitivity to the reciprocity feedback, with unforgiving being the highest in sensitivity and cooperative being the lowest. The remaining strategies of random and defective are insensitive to reciprocity.

\section{Parametric Set-Up}

For modeling and simulating our framework, we used NetLogo which is a multi-agent programmable modeling environment. NetLogo was designed for multiple audiences in

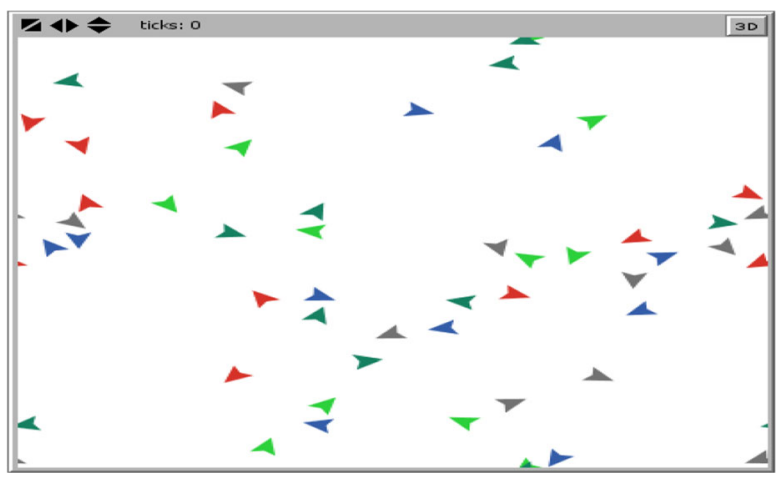

Fig. 2 Randomly distributed players on a landscape of $130 \times 130$ cells 
mind, especially when involving domain experts without a programming background to model-related phenomena. In our simulation, the initial knowledge of each player is assigned an arbitrary value according to $\operatorname{rand}(200,300)$. All of this knowledge is assumed to be transferrable. The synergy value $\mathrm{kg}_{\text {fus }}$ for each player is assumed to be a random number from a uniform distribution in the interval [10, 30]. $\mathrm{kg}_{A \leftarrow B}^{A}$ is assumed to be uniformly distributed random number in the interval $[5,20] . \mathrm{kg}_{c p t v}^{A}$ is assumed to be $0.25^{*}\left(\sigma_{B} k g_{T}^{A}+k g_{f u s}^{B}+k g_{B \leftarrow A}^{B}\right) / \sigma_{B} k g_{T}^{A}$. The same is true for the opponent player. The capacity for each player to absorb knowledge $(\sigma)$ is assumed to be 0.9 . The initial trust $\left(T_{0}\right)$ for each individual is assumed to take values that are uniformly distributed in the interval $[500,600]$. The penalty for hoarding $(\lambda)$ is assumed to be 5 units.

\section{Analysis of Results}

Several scenarios have been setup for simulation. For each of the simulated scenarios in the paper, we reported the average and standard deviation of the logarithm of summation of knowledge of all players in a certain strategy $\left(\sum \mathrm{kg}_{s}\right)$, divided by total knowledge of all players in all strategies $\left(\log \left(\frac{\sum \mathrm{kg}_{s}}{K G_{T}}\right)\right.$. We used enough number of iterations for developing the agents and their skills, and that is sufficient to describe the trend and behavior of all involved strategies. This is reported for a number of iterations enough to describe the trend and behavior of the strategy over long period of time. In the first scenario, all strategies have equal number of players, that is, 20 players. We thought of this formation as a fair initial reference situation. Each player is initialized with values selected in the manner that was previously described. Figure 3 shows a sample run, where after about 50 iterations, the trend of each strategy became apparent. The three strategies of cooperative, tit-for-tat, and unforgiving seem to mutually increase at the first period of time against a decay for the other two strategies. After the strategies of defective and random saturate, the cooperative strategy takes off faster than its two temporary companions. The experiment was repeated for 25 independent runs with each strategy having 20 players, and the number of iterations was increased to 5000 . Figure 4 shows the averaged percentage of knowledge for each strategy.

The two strategies of defective and random were stabilized (apparently decline) over a period of 2000 iterations. The saturation could be explained by two causes. Firstly,

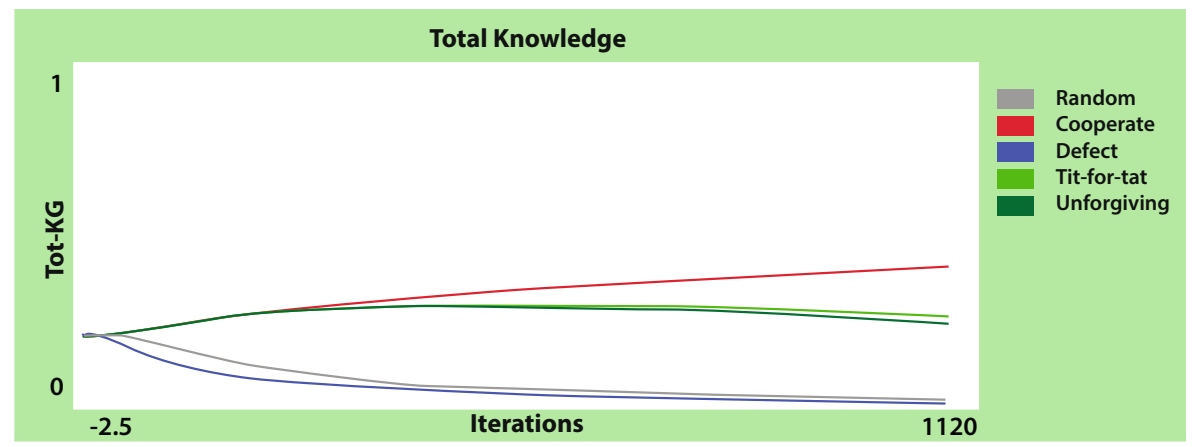

Fig. 3 Percentage of knowledge for each strategy over 1120 iterations with 20 affiliates for each strategy 


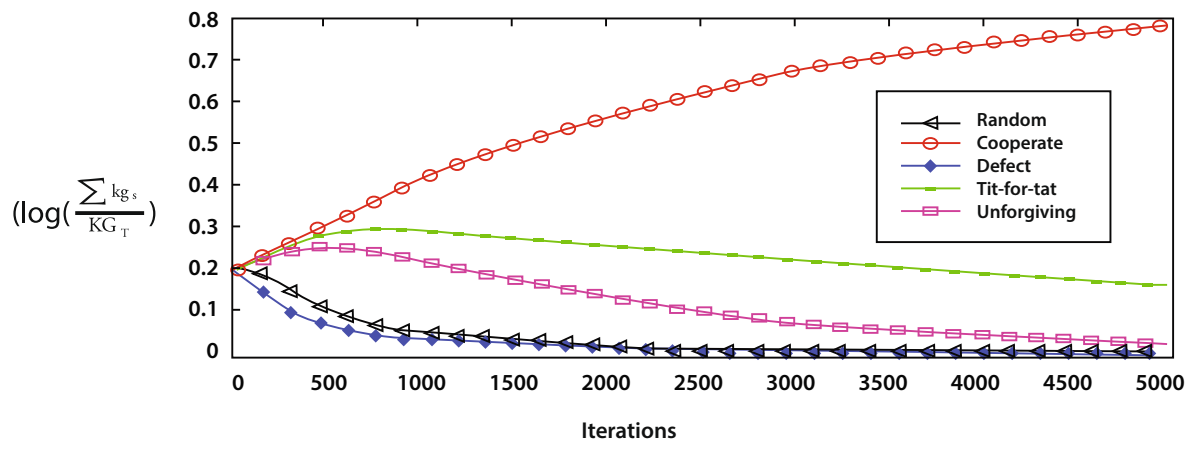

Fig. 4 Percentage of knowledge for each strategy over 5000 iterations, averaged over 25 independent runs with 20 affiliates for each strategy

the unforgiving players (20\% of players) will stop transferring knowledge to the defectors immediately after only one iteration and after one defection incident from a random-strategist player. A tit-for-tat player will exhibit a similar behavior towards the defector players. However, a tit-for-tat player will simply repeat the decisions of a random player one iteration later. Secondly, the main source of knowledge for the defectors and random players would be the cooperative players. Yet, this source will dry out when the trustworthiness of these two strategies drops below the threshold level $\left(T_{t h}\right)$.

The cooperative strategy gains knowledge at a rate close to the two strategies of titfor-tat and the unforgiving but then takes off at a higher rate. A cooperative player does not gain knowledge only when meeting a defector or in some random cases, which explains the period of slow rate of knowledge gain. When these two strategies turn untrusted and thus saturate, the cooperative players will have more chances of knowledge gain. This explains the acceleration in knowledge gain for cooperative players. The same case applies to the two strategies of tit-for-tat and unforgiving. However, the strategies of these two groups, unlike the cooperative, are reciprocity dependent. This dependence drives them to act as defectors in some cases which hurts their knowledge gaining opportunities in a fashion equal to the cooperative. The knowledge of these two strategies would thus saturate after a period of time. The four curves appear declining because we are representing a strategy's gain as a ratio of the total gain. The high rate of knowledge acquisition within the cooperative strategy makes the other strategies look declining. It is worth noting that after 5000 iterations, the collective knowledge is $80 \%$ comprised of knowledge that resides in cooperative players. In other words, we will have two equilibrium situations: one for cooperative players and another for the rest. Interestingly, 20 individuals are contributing $80 \%$ of the collective knowledge, while 80 individuals are contributing only $20 \%$.

In the second simulation, we gave advantage to the defective strategy following the common wisdom that promotes hoarding, "knowledge is power." The defective strategy is assigned 50 players, while every other strategy is assigned 20 players. The results revealed that this modification turned the random strategy into the worst strategy (Fig. 5). It is the collective knowledge of the higher number of players that gave the defectors an advantage over random players. It takes larger number of KS rounds to surpass the level of knowledge of 50 defective players. The performance of the two 


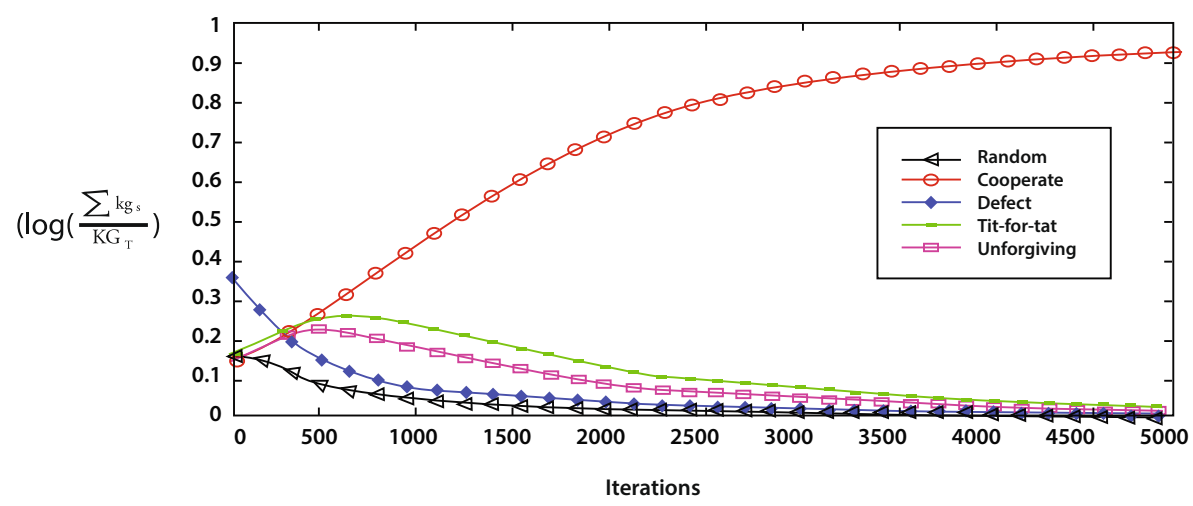

Fig. 5 Percentage of knowledge for each strategy over 5000 iterations, averaged over 25 independent runs with 50 affiliates of defective strategy and 20 affiliates for every other

strategies tit-for-tat and unforgiving demonstrates faster decline with the increase of number of defectors. This is an interesting observation which supports literature that described knowledge hoarders as a negative influence on the work environment (Karkoulian et al. 2013; Sandhu and Ching 2014). Actually, the long-run performance of the three strategies with initial sharing intention goes in congruence with their level of sensitivity to defection. The lower the sensitivity, the better the performance over the long-run. The low sensitivity of cooperative players towards defective behavior brings the highest knowledge gain. The faster stabilization of the level of knowledge gain for the players with other strategies makes the relative share of cooperative players even higher, $90 \%$ of collective knowledge.

In the second scenario, we have increased the affiliates of the extreme strategy of defection. This gave defectors advantage over the short run, which they lost over the long run. It was obvious that giving the same lead to cooperative players would give them an advantage that they would permanently maintain. Accordingly, we decided to attempt a third scenario with the lead given to a strategy with a middle sensitivity to reciprocity. We assigned 50 players to the tit-for-tat strategy and 20 players for each of the others. As Fig. 6 shows, the higher number of tit-for-tat players prolonged the time before saturation for this strategy is reached. It took around 1000 iterations to see a clear decreasing trend in the curve that describes the percentage knowledge acquired by titfor-tat affiliates. Additionally, it took over 4000 iterations to lower the knowledge contribution of this group below $40 \%$. It is worth noting that higher number of tit-fortat players gave cooperative players a better opportunity to increase their knowledge. This is because a tit-for-tat player behaves exactly as a cooperative player when the two meet. It is the two strategies of defective and random that erode the high contribution of tit-for-tats.

Finally, we calculated the mean and standard deviation for each strategy in each scenario (Table 5). The values show that the cooperative strategy has the highest mean. Accordingly, we tested the statistical significance between the mean of each strategy as compared with the cooperative mean. The test was based on a $t$ test with $95 \%$ confidence level. As an indication of results, we use a "+" if there is statistical significance difference, "." if there is no difference, and "N/A" if the group is the base 


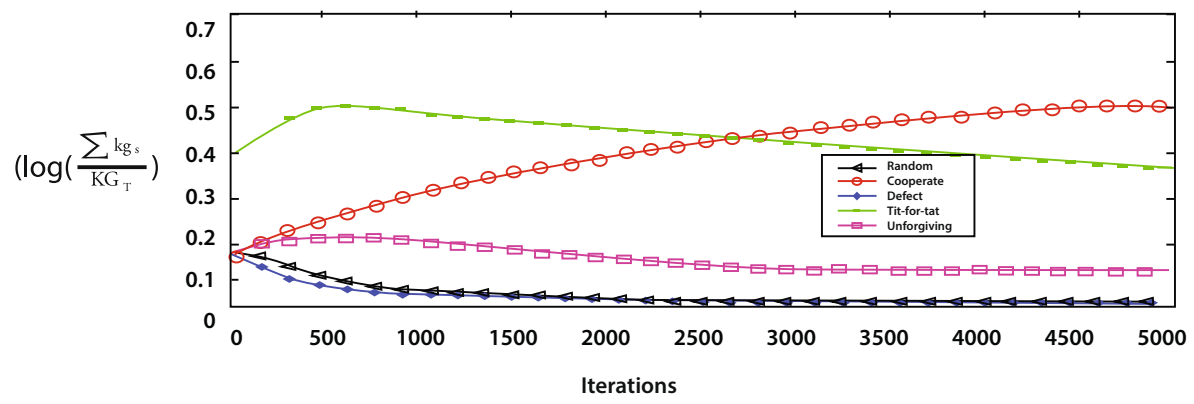

Fig. 6 Percentage of knowledge for each strategy over 5000 iterations, averaged over 25 independent runs with 50 affiliates of tit-for-tat strategy and 20 affiliates for every other

for comparison (best value). All differences proved to be statistically significant as shown in Table 5.

\section{Discussion and Conclusions}

The failure rate in knowledge management projects is anticipated to be $50 \%$ ( $\mathrm{Li}$ and Jhang-Li 2010). According to the authors, the lack of proper KS incentives system is one cause. Many studies have addressed the KS behavior in an attempt to understand the factors affecting it. Despite all the studies, we believe KS behavior needs further examination. For this purpose, we developed a KS framework with a game-theoretic perspective. The framework embraces conclusions from other research, especially the effect of intrinsic factors such as enjoyment, and extrinsic factors such as reciprocity.

Several studies have identified the game theory as an appropriate problem framing technique that would provide useful insights (Mueller et al. 2005; Sharma and

Table 5 Statistical significance of mean value comparisons for each of the three scenarios

\begin{tabular}{llllll}
\multicolumn{2}{l}{ Scenario (1). Each strategy starts with 20 individuals } & & & \\
Strategy & Random & Cooperative & Defective & Tit-for-tat & Unforgiving \\
Mean & 0.01277 & 0.4804 & 0.0109 & 0.2751 & 0.25815 \\
(Std. Dev.) & $(0.00417)$ & $(0.06467)$ & $(0.00194)$ & $(0.01171)$ & $(0.01371)$ \\
Statistical significance & + & N/A & + & + & +
\end{tabular}

Scenario (2). Defective strategy starts with 50 individuals. Every other strategy starts with 20 individuals

$\begin{array}{llllll}\text { Strategy } & \text { Random } & \text { Cooperate } & \text { Defect } & \text { Tit-for-tat } & \text { Unforgiving } \\ \text { Mean } & 0.05098 & 0.57206 & 0.08356 & 0.15706 & 0.2218 \\ \text { (Std. Dev.) } & (0.00382) & (0.02611) & (0.00825) & (0.00636) & (0.01398) \\ \text { Statistical significance } & + & \text { N/A } & + & + & +\end{array}$

Scenario (3). Tit-for-tat strategy starts with 50 individuals. Every other strategy starts with 20 individuals

\begin{tabular}{llllll} 
Strategy & Random & Cooperate & Defect & Tit-for-tat & Unforgiving \\
Mean & 0.0000 & 0.47852 & 0.0000 & 0.40978 & 0.16442 \\
(Std. Dev.) & $(0.0000)$ & $(0.02755)$ & $(0.0000)$ & $(0.01379)$ & $(0.04414)$ \\
Statistical significance & + & N/A & + & + & + \\
\hline
\end{tabular}


Bhattacharya 2013; Liu et al. 2019). The rational action theory explains how the players in a game are guided by their perception of utility. Similar to previous attempts (Wang et al. 2014), we have constructed a utility functions. Yet, unlike other studies, we limited the exchanged utility units to only three types: knowledge, competitiveness, and trustworthiness. The exclusion of organizational rewards allows us to understand the natural KS behavior without any organizational intervention, baseline KS behavior. Another improvement to previous studies is the usage of the assurance game instead of the Prisoner's Dilemma game. Earlier game-theoretic approaches that used the Prisoner's Dilemma game found mismatching results when compared with empirical studies (Mueller et al. 2005). This was due to the fact that a Prisoner's Dilemma is a one-round game and does not represent the notion of reciprocity. Our proposed framework uses the assurance game which is a multi-round game. Our trustworthiness matrix allows the implementation of this type of games. The final major improvement in our proposed framework is the introduction of the notion of KS strategies to represent individuals' sharing behavior instead of KS intention. We identified five KS strategies and explained how these strategies guide the dynamics of KS intention.

Our goal was to observe and compare the KS behavior of each type of strategy. We simulated three scenarios, where a scenario is a collection of players from all five strategies getting engaged in several rounds of KS opportunities. In the first scenario, we assigned 20 players to each strategy. The simulator calculated the gained knowledge of each strategy as a percentage of the total gained knowledge. The graph showed an immediate divergence between cooperative, tit-for-tat, and unforgiving as one group and defective and random as a second group, with an advantage for the former group. This means that in a working environment with low number of defective individuals, these people would find themselves slowly deprived of receiving knowledge. At a later time, another divergence takes place between cooperative as a group and tit-for-tat and unforgiving as a second group, with an advantage for the former. This is due to the lower sensitivity of the cooperative towards hoarding behavior.

In the second scenario, we simulated a situation where number of individuals with initial sharing intention is equal to the number of individuals with hoarding intention. We assigned 50 individuals to the defective strategy and 20 individuals to each of the others. The results show that their high number gave defectors an edge over all other strategies for about 500 rounds. The other strategies immediately encounter a divergence leaving the random strategy unaccompanied with the poorest performance. The second divergence occurs at 500 rounds leading to three paths, with cooperative strategists taking the superior path alone. A moderate performance path is taken by both tit-for-tat and unforgiving strategists. The remaining strategies are taking the path with the lowest performance. So, the high number of defectors altered the relative performances on the short run. This alteration could be explained by the different sensitivity towards the hoarding behavior and to the increase in the number of individuals with this intention. This initial superiority of the defective strategy could explain the belief that hoarding is power.

In the third scenario, we increased the number of individuals with moderate level of sensitivity. We assigned 50 individuals to the tit-for-tat strategy and 20 individuals to each of the others. Again, their high number gave the tit-for-taters an initial edge, yet for a larger number of rounds than the defectors enjoyed, almost 3000 rounds. The 
cooperative players had to wait this long for the trustworthiness of the tit-for-taters to erode. Also in this scenario, the defective and random players behave as a group in diverging themselves immediately from the cooperatives and the unforgiving. After 500 rounds, the cooperatives and the unforgiving diverge with an advantage for the cooperative.

In conclusion, the simulations reveal a short-run performance of the different strategies that is highly influenced by the number of strategy affiliates. This is in accord with previous literature on the effect of cultural values in creating KS intention and the negative effect of knowledge hoards on KS behavior. However, the simulation adds value by revealing the long-run performance. Regardless of the relative numbers of affiliates, the KS behavior will eventually settle in two equilibria situations. All cooperative players will settle alone in one situation and would be contributing the highest share to collective knowledge. All other strategists will converge into the second equilibrium situation and would be of low contribution to collective knowledge. This baseline understanding of the KS behavior is of value to the organization. The managers should shift their attention from the notion of KS intention to the notion of KS strategy. Both strategies of tit-for-tat and unforgiving start with a positive KS intention, yet both of them end up settling with the defective individuals.

\section{Empirical Study}

\section{Study Design}

In an attempt to verify the simulated KS behavior, we intended to carry out an empirical study at a local hospital. Hospitals are exemplary environments for studying KS practices. The human nature of the medical services and the fact that physicians are sworn to perform at their best should provide a natural motivation for positive KS intentions. Unfortunately, the current emergency situation caused by the COVID-19 pandemic has made the hospital's attention to non-medical research of low priority. We had to design a study with a narrow scope that would fit the limited attention that we would get from the overwhelmed physicians. The targeted hospital has a size of 650 beds with about 200 specialists and 400 resident physicians. The residents are medicine school graduates who are yearly admitted in a 4-6 years residency program. The residents could specialize in one of 15 possible medical areas. The possibility of KS among the residents is higher when they are in the same year and the same specialization.

A longitudinal study that target residential doctors at their first year in only one specialization would be the best design for our KS framework verification. The study would attempt to identify the residents' strategies and assess their initial KS intention, trustworthiness, and level of knowledge and would track the changes along time and experience. Unfortunately, such a study would have to be delayed to a future time. Instead, we attempted a concise and preliminary study with narrower scope. The basis of our study is still related to our proposed KS framework as shown in Fig. 7.

We attempted to collect two assessments of each of level of KS intention, level of professional obligation, level of belief in effect of KS on performance, and level of trustworthiness. The empirical data were to be used for: 


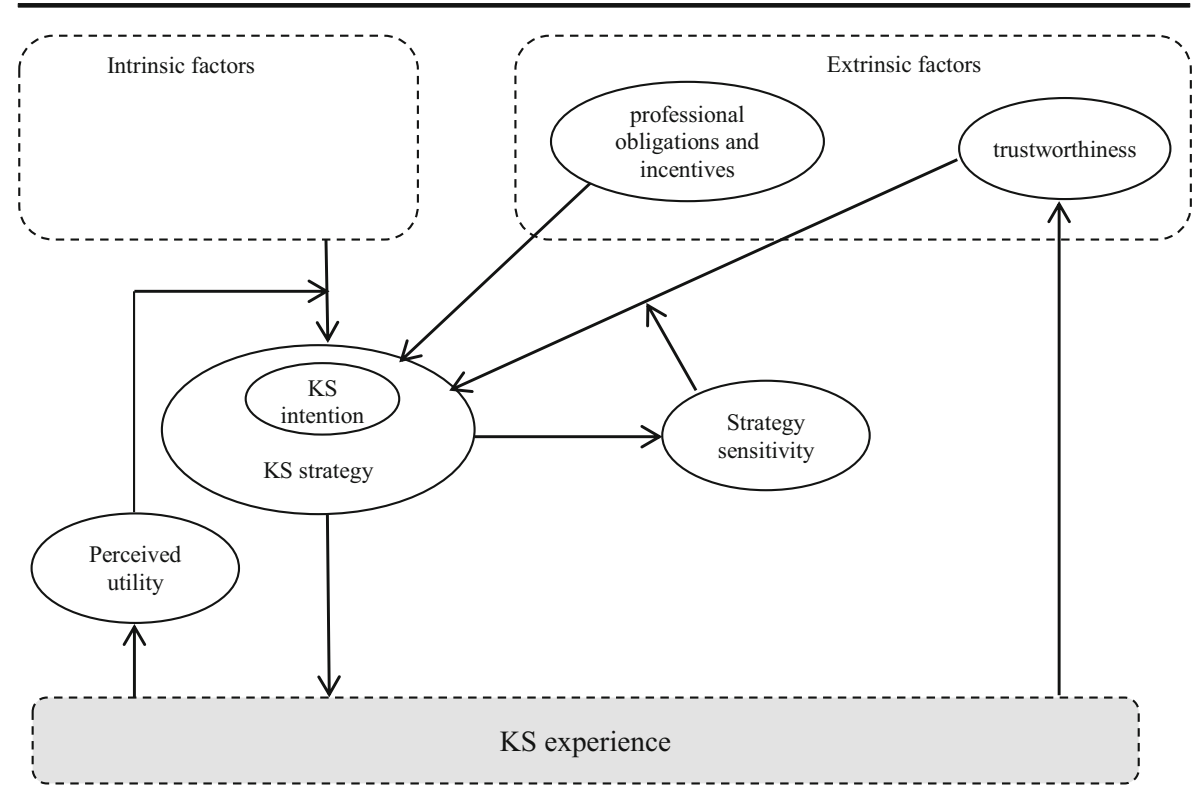

Fig. 7 The empirical study model

1. Verifying the residents' perceived utility of $\mathrm{KS}$ on performance

2. Verifying a divergence in $\mathrm{KS}$ intention along two trends towards two equilibria situations

3. Studying the effect of professional obligation as an extrinsic factor on KS intention

4. Verifying a decreasing trend in level of trustworthiness over KS experiences

5. Verifying an increasing sensitivity towards decreasing trustworthiness over KS experiences

We designed a concise survey with only 9 questions to collect our data. Four questions targeted the residents' initial perceptions, and five questions targeted their current perception. We used a descriptive 6-level Likert scale as shown in Fig. 8, with each level worth 1 unit for analysis purposes. We distributed the survey electronically to 204 specialists and 400 residents. Despite the required 5-min completion time, only 12 specialist physicians completed the survey, that is, $4 \%$ response rate. Residents' response was relatively better and reached 70 responses, $18 \%$ response rate. Unfortunately, we had to discard the responses from the specialists and limit the analysis to residents' responses.

\section{Perceived Utility of KS on Performance}

We started the analysis by checking the residents' perception of the effect of KS on their personal performance and organizational performance. As Table 6 shows, the overall perception in the effect of KS on both personal and organizational performances was comparable around $70 \%$. The changes in these perceived utilities from this initial state to the current state were insignificant. The residents consistently over time perceived KS as supportive of their personal performance as well as the organizational 
1- How would you rate your current level of personal motivation to share your knowledge with colleagues?

\begin{tabular}{|l|l|l|l|l|l|}
\hline Extremely motivated & Very motivated & motivated & reluctant & Very reluctant & Extremely reluctant \\
\hline
\end{tabular}

2- How would you rate your current level of sense of professional obligation/incentives to share your knowledge with colleagues?

\begin{tabular}{|c|c|c|c|c|c|}
\hline $\begin{array}{c}\text { Extremely obliged } \\
\text { with incentives }\end{array}$ & $\begin{array}{c}\text { Obliged with } \\
\text { incentives }\end{array}$ & obliged & Low obligation & $\begin{array}{c}\text { Low obligation with } \\
\text { no incentives }\end{array}$ & $\begin{array}{c}\text { Extremely low } \\
\text { obligation with no } \\
\text { incentives }\end{array}$ \\
\hline
\end{tabular}

3- How would you rate your current expectation of the percentage of colleagues with positive attitude towards sharing their knowledge with you?

\begin{tabular}{|c|c|c|c|c|c|c|c|c|c|c|}
\hline $100 \%$ & $90 \%$ & $80 \%$ & $70 \%$ & $60 \%$ & $50 \%$ & $40 \%$ & $30 \%$ & $20 \%$ & $10 \%$ \\
\hline
\end{tabular}

4- How would you rate your current level of confidence that a collective knowledge sharing behavior improves your PERSONAL performance?

\begin{tabular}{|c|c|c|c|c|c|}
\hline Extremely confident & Very confident & confident & doubtful & Very doubtful & Extremely doubtful \\
\hline
\end{tabular}

5- How would you rate your current level of confidence that a collective knowledge sharing behavior improves ORGANIZATIONAL healthcare performance?

\begin{tabular}{|l|l|l|l|l|l|}
\hline Extremely confident & Very confident & confident & doubtful & Very doubtful & Extremely doubtful \\
\hline
\end{tabular}

Fig. 8 The empirical study survey questions for collecting residents' current perception

performance. Interestingly, the individuals with initial hoarding intentions (4 individuals) had a 33\% increase in their perception of effect of KS on personal performance and $92 \%$ increase in their belief of the effect of KS on organizational performance. However, the small sample does not allow a significance test and could only hint for further investigation.

\section{The Trends in KS Intention}

Our assessment of KS intention was not based on a dichotomous approach, either a sharing intention or a hoarding intention. Instead, we allowed the participants to express their KS intentions on a 6-level Likert scale, with no neutral state. The residents were asked to rate both their initial KS intentions and their current KS intentions. The latter represents the KS intention after medical practice provides feedbacks on trustworthiness. Figure 9 shows the percentages of the 70 responses over the 6 levels of KS intentions at both the initial state and the current state. Table 7 shows the changes in KS intentions. The numbers reveal two trends: firstly, an increase in number of hoarders

Table 6 Level of perception of effect of KS on personal and organizational performances

Average collective perception of KS effect on personal performance

\begin{tabular}{|c|c|c|c|c|c|c|c|}
\hline $\begin{array}{l}\text { Initial } \\
\text { level }\end{array}$ & $\begin{array}{l}\text { Current } \\
\text { level }\end{array}$ & $\begin{array}{l}\text { Change in } \\
\text { level }\end{array}$ & $\begin{array}{l}\text { Statistical } \\
\text { significance }\end{array}$ & $\begin{array}{l}\text { Initial } \\
\text { level }\end{array}$ & $\begin{array}{l}\text { Current } \\
\text { level }\end{array}$ & $\begin{array}{l}\text { Change in } \\
\text { level }\end{array}$ & $\begin{array}{l}\text { Statistical } \\
\text { significance }\end{array}$ \\
\hline $66 \%$ & $72 \%$ & $6 \%$ & $p=0.3463$ & $68 \%$ & $70 \%$ & $2 \%$ & $p=0.7939$ \\
\hline
\end{tabular}

Average collective perception of KS effect on organizational performance 
and secondly a decrease in number of sharers. Since the number of initial hoarders is small, the percentage increase in number of hoarders is higher the percentage decrease in number of sharers.

Our theoretical proposed framework assumed the KS behavior to follow an assurance game. This was evident in the simulations when all scenarios revealed a divergence in the gained knowledge leading to two trends and settling in two equilibria states. The two trends in gained knowledge were caused by a divergence in the KS intention. Our empirical data verifies the existence of these two trends in KS intention. The statistical test for the significance of the change in the level of sharing intentions showed that the change was significant as indicated by the calculated $p$ value. The change in the level of hoarding intention was also found to be significant.

We identified 66 residents with initial sharing intentions comulating a sharing value of 125 units. Out of these 66 residents, 17 residents had a drop in their initial KS intention with a total value of 47 units. Figure 10 shows the trending decrease in KS intention. On the other hand, 49 residents with total KS intention value of 78 units maintained, or increased, their initial KS intention. Figure 11 shows the trending among the residents who maintained or increased their initial hoarding intention. In conformity with the simulations, the group of initial knowledge sharers is diverged into two groups: one maintaining their initial sharing intention (supposedly the cooperative strategists) and another converting and joining the initial knowledge hoarders (supposedly the tit-for-tats and unforgiving strategists). Unfortunately, this study cannot confirm that the cooperative strategists are the group who are maintaining their stands, while the tit-for-tats and the unforgiving strategists are the ones who are converting. This verification has to be addressed in a future study.

\section{Effect of Professional Obligations}

Our hospital does not apply a KS reward system, which eliminates the effect of this extrinsic motivation. However, we assumed that medical physicians would have their distinctive professional motivations dictated by the nature of healthcare services. We attempted to measure such motivations before and after the residential experience. Table 8 shows the changes in level of perception of KS professional obligation and its parallel changes in KS intentions.

It seems that the practice of medicine has a great effect on residents' perception of KS professional obligations causing it to increase by $30 \%$. This increase was

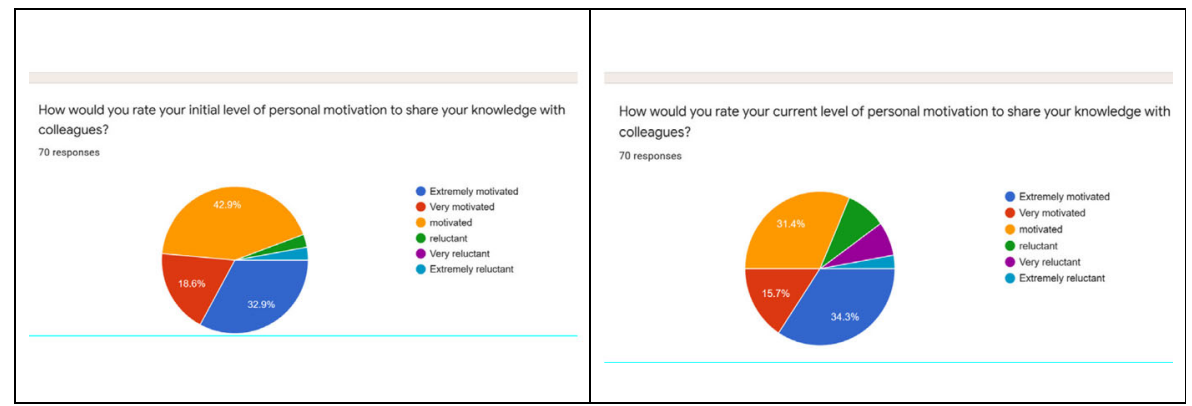

Fig. 9 The initial and current percentages of each level of KS intention 
Table 7 Changes in KS intentions

Collective sharing intentions

\begin{tabular}{llllllll}
\hline $\begin{array}{l}\text { Initial } \\
\text { level }\end{array}$ & $\begin{array}{l}\text { Current } \\
\text { level }\end{array}$ & $\begin{array}{l}\text { Percentage } \\
\text { change } \\
\text { in level }\end{array}$ & $\begin{array}{l}\text { Statistical } \\
\text { significance }\end{array}$ & $\begin{array}{l}\text { Initial } \\
\text { level }\end{array}$ & $\begin{array}{l}\text { Current } \\
\text { level }\end{array}$ & $\begin{array}{l}\text { Percentage } \\
\text { Change in } \\
\text { level }\end{array}$ & $\begin{array}{l}\text { Statistical } \\
\text { significance }\end{array}$ \\
\hline $94.4 \%$ & $81.4 \%$ & $-13.8 \%$ & $p=0.4512$ & $5.6 \%$ & $18.6 \%$ & $232 \%$ & $p=0.1711$ \\
\hline
\end{tabular}

statistically tested to be significant. Yet, instead of an expected increase in KS intentions, we observed an $11 \%$ decrease which was statistically insignificant. The high level in belief that KS enhances performance accompanied by a significant increase in the professional obligation to share knowledge has not caused a change in $\mathrm{KS}$ intention. A possible explanation is a negative influence excreted by a decrease in trustworthiness. This latter effect is to be tested in the next sub-section.

\section{Effect of Trustworthiness}

Our sample was composed of 70 residents admitted in 6 levels of the medical residency program. The survey asked each resident to rate his initial KS intention. However, when rating initial level of trustworthiness, the survey did not ask a direct question. Instead we preferred to infer the initial level of trustworthiness from the average initial KS intention of each level of residents. Hence, each level of residents starts with one value of initial trustworthiness that is their collective initial willingness to share knowledge. The KS experiences which residents go through would affect this initial value causing it to supposedly decay over time. Our simulation revealed that the percentage of individuals with defective strategy is the cause of trustworthiness decay. Additionally, their relative number decides the decay rate. Based on the expressed initial KS intention, our survey results could identify those with defective strategy. This group was identified to comprise $5.6 \%$ of the surveyed residents. It is the KS experience against this group that would erode each resident's level of trustworthiness. We calculated the change in trustworthiness of each resident and identified a total decrease

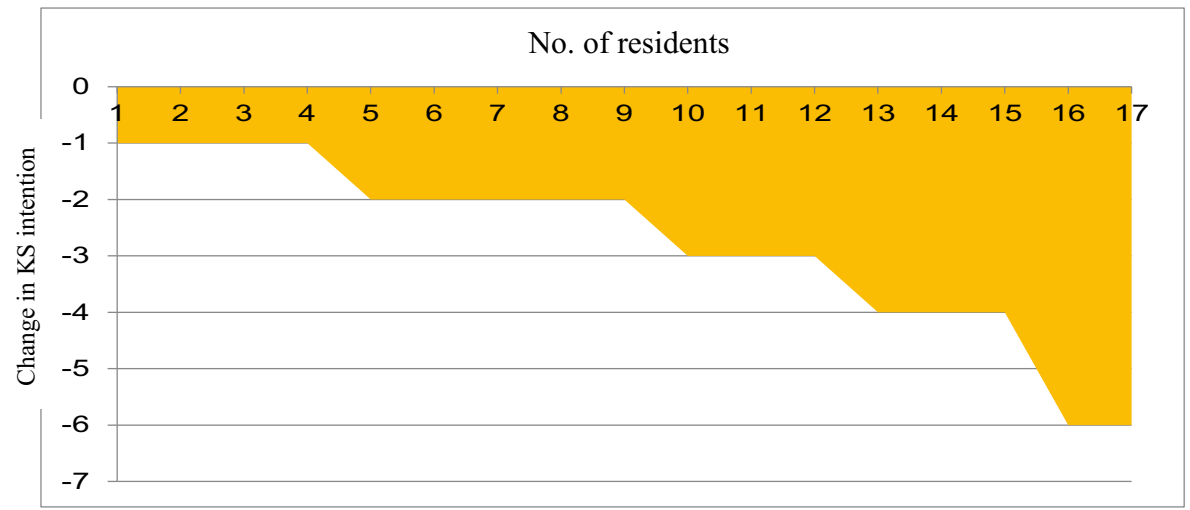

Fig. 10 The trend of divergence from a sharing intention to hoarding intention by 17 residents 


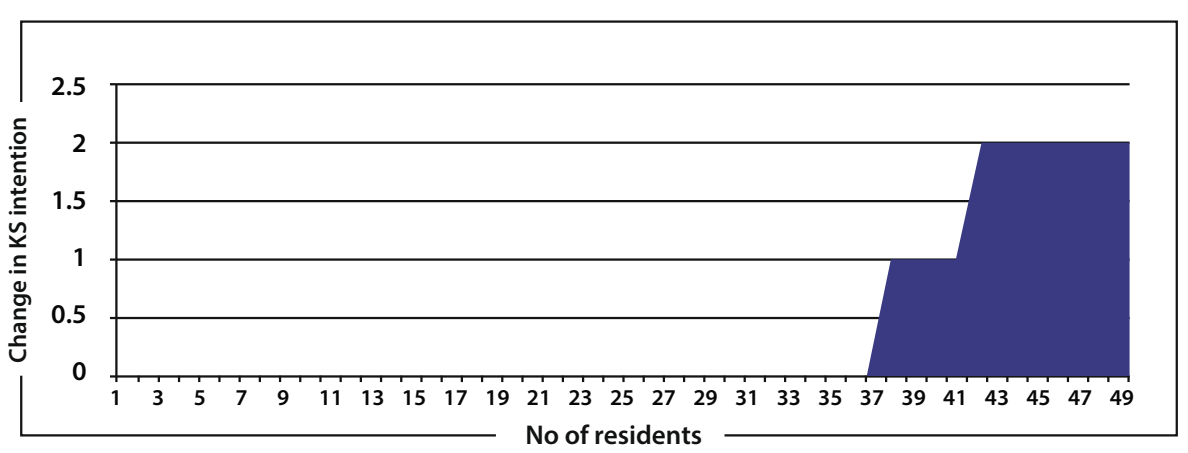

Fig. 11 The trend of maintaining or increasing the sharing intention by 49 residents

by an amount of 167 units, $88.4 \%$ of the changes. On the other hand, we identified 22 units of increase in trustworthiness that accounts for $11.6 \%$ of the changes. Figure 12 shows the distributions of these changes along with 4 cases that maintained their level of trustworthiness.

Figure 13 shows the decrease in trustworthiness across the 6 levels of residents. Most levels revealed an average of lower trustworthiness following their KS experiences. The lack of a pattern could be explained by having different combinations of strategies across the yearly admitted residents. As our simulator have revealed, each combination of strategies would result in different sensitivity to defection, thus to different decay rate of trustworthiness.

\section{Effect of Sensitivity of KS Strategies}

We have previously explained how KS strategies that start with a sharing intention have different sensitivity towards a defective opponent. The unforgiving has the highest sensitivity with one defection sufficient to cause the sharer's conversion without any chance for redemption. The tit-for-tat is similar in its reaction for the first defection, yet it allows the defector to redeem himself. Finally, the cooperative player acts in a similar fashion as a tit-for-tat player but with higher patience in terms of number of tolerated defections. Instead of one tolerated defection, the cooperative waits for the trustworthiness threshold to fall below $T_{\mathrm{th}}$. The collective sensitivity of the KS intention is then dependent on the combination of the individuals and their strategies, the higher number of unforgiving players, the higher the collective sensitivity. The higher the number of cooperative players, the lower the collective sensitivity.

Table 8 Changes in level of perception of KS professional obligation and in KS intentions

Change in KS professional obligation

\begin{tabular}{lllllllll}
\hline $\begin{array}{l}\text { Average } \\
\text { initial } \\
\text { level }\end{array}$ & $\begin{array}{l}\text { Average } \\
\text { current } \\
\text { level }\end{array}$ & $\begin{array}{l}\text { Change } \\
\text { in level }\end{array}$ & $\begin{array}{l}\text { Statistical } \\
\text { significance }\end{array}$ & $\begin{array}{l}\text { Average } \\
\text { initial } \\
\text { level }\end{array}$ & $\begin{array}{l}\text { Average } \\
\text { current } \\
\text { level }\end{array}$ & $\begin{array}{l}\text { Change } \\
\text { in level }\end{array}$ & $\begin{array}{l}\text { Statistical } \\
\text { significance }\end{array}$ \\
\hline $2 \%$ & $32 \%$ & $30 \%$ & $p=0.00934072$ & $56 \%$ & $45 \%$ & $-11 \%$ & $p=0.199276255$ \\
\hline
\end{tabular}

Change in KS intention 


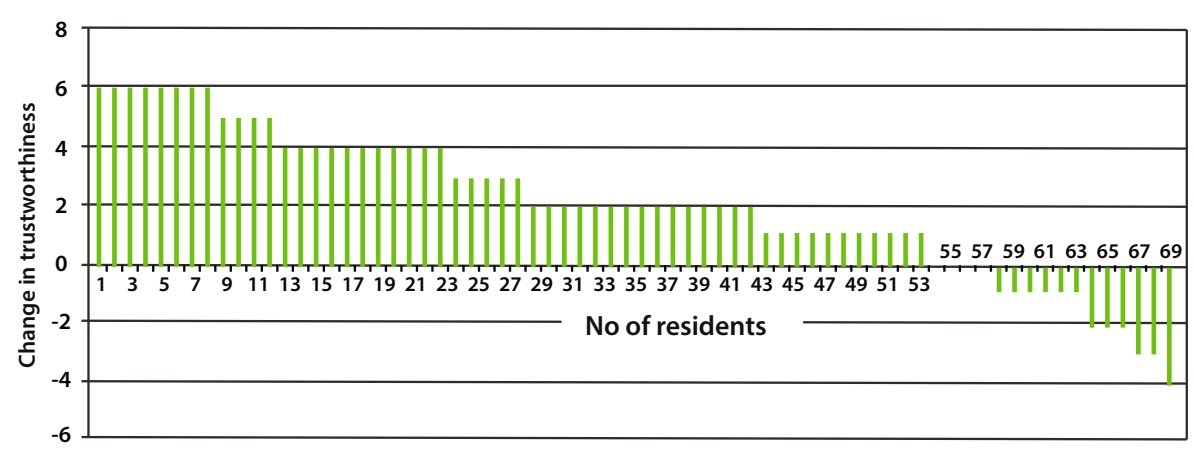

Fig. 12 Distribution of the changes in trustworthiness across all residents

Moreover, an organizational environment with only cooperative, tit-for-tat, and unforgiving strategists would demonstrate the exact behavior as an environment with complete cooperative strategists. It is the numbers of random and defector strategists who triggers the conversion behavior of the KS system. This behavior was evident in our simulations. The previous argument leads to concluding that junior residents should have lower sensitivity towards decreasing trustworthiness than senior residents, lower chance of reaching $T_{\text {th }}$. We divided our 70-resident sample into two groups: a junior group of first and second year residents and a senior group of $\mathbf{3}$ years and above, the former comprised 36 residents, while the latter comprised 34 residents. Table 9 reveals the sensitivity for junior residents as compared to the sensitivity of senior residents.

As noted, there was a $37 \%$ decrease in level of trustworthiness among junior residents, which was statistically a very significant drop. Yet, this drop caused only $18.9 \%$ decrease in KS intention, which was statistically insignificant. Moreover, the calculated correlation factor of .053 indicates no correlation between trustworthiness and KS intention among junior residents. On the other hand, a similar decrease in trustworthiness among senior residents (33\%) caused a $41.5 \%$ decrease in KS intention. The statistical testing showed that this latter decrease was still insignificant. However, it

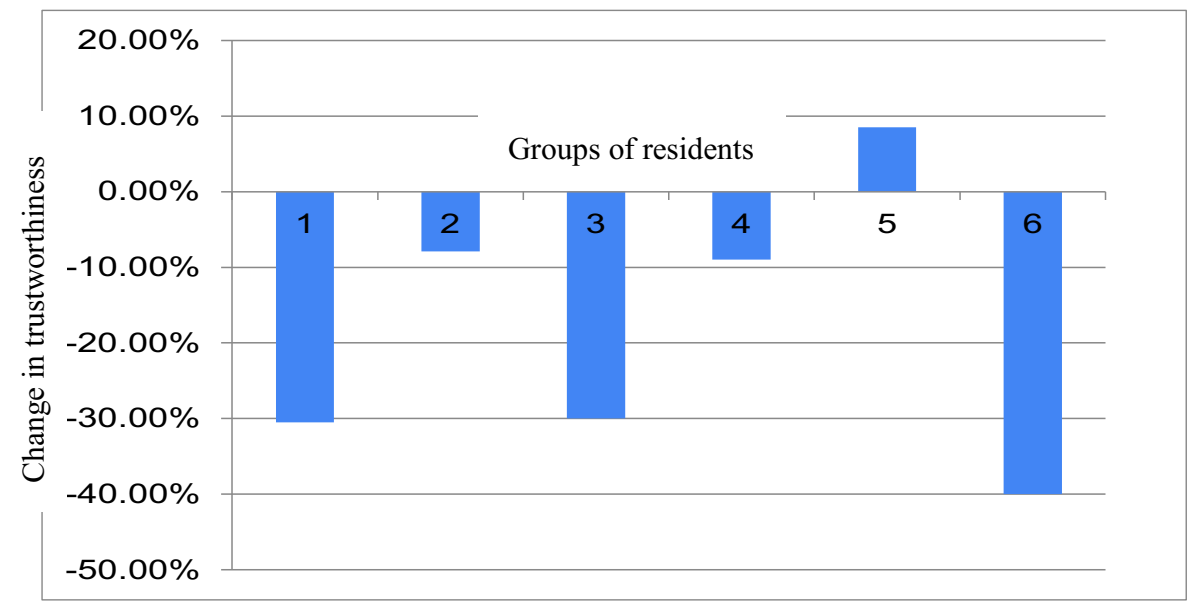

Fig. 13 Level of decrease in trustworthiness across the 6 levels of residents 


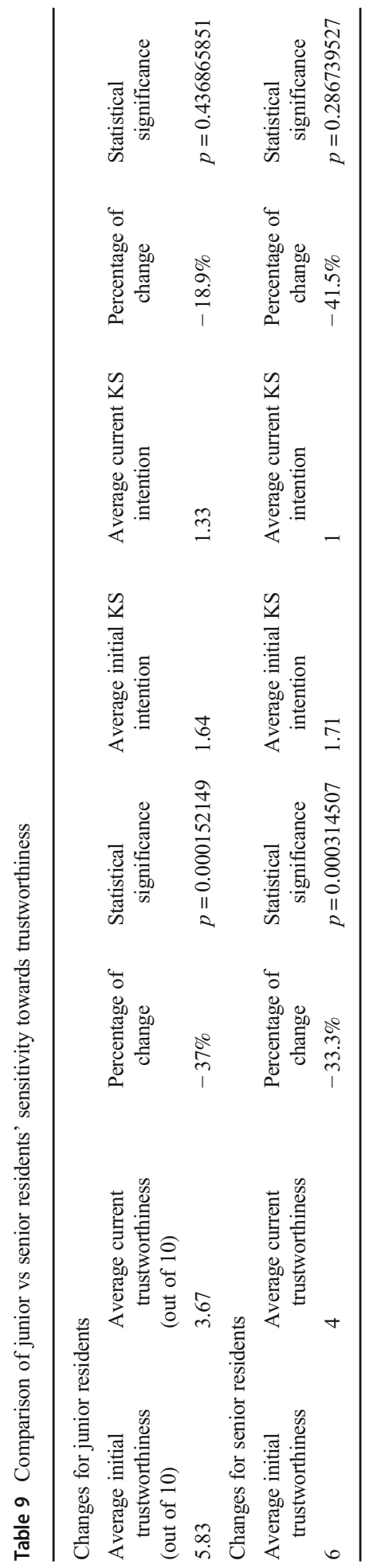


is the change in the $p$ value that reveals the trend; the $p$ value has dropped by almost $35 \%$ indicating a trend of increasing sensitivity. Also, the correlation factor increased significantly from .053 to .45 .

\section{Significance of Study}

This study has provided a framework for KS behavior using a game-theoretic approach. The basis of the KS behavior is the individual's perception of utility. Our tested framework has limited the utility to only three types: knowledge, competitiveness, and trustworthiness. These units of utility are traded in every KS activity based on an instantaneous sharing/hoarding decision. These decisions are operational decisions within a wider decision strategy. Previous KS studies and practices have been highlighting the concept of sharing intentions and the intrinsic and extrinsic factors affecting it. Organizational rewarding for sharing intentions was highly investigated.

We have demonstrated through simulations and empirical data that initial sharing intentions are necessary but not sufficient to understand the dynamics of KS behavior. We argued for the adoption of the notion of KS strategies as an incubator of KS intention. We supported our argument with simulations and empirical results. Some individuals start with sharing intentions but with higher sensitivity to opponents' hoarding behavior. Such sensitivity shortens the lifetime of the initial sharing intention causing divergence towards a hoarding equilibrium situation. The mechanisms of the different KS strategies explain such behavior. Accordingly, research attention should shift to theoretical and practical examination of individuals' KS strategies rather than KS intentions.

We have developed a KS framework that supports the theoretical exploration of many KS organizational situations. Our testing of the framework was limited in terms of the number of introduced intrinsic and extrinsic factors affecting KS strategy. Future studies would manipulate the setup of the proposed framework introducing several possible combinations of intrinsic and extrinsic factors. Also, our testing monitored only the progression of individuals' knowledge over a series of KS experiences. Future studies would consider other outcomes such as competitiveness and trustworthiness. The behavior of these outcomes should be associated with the types of strategies, which would have a great impact on individuals' recruitment and performance appraisal. On top of all, sufficient empirical studies need to be carried out to extendedly verify the framework and its embedded utility functions.

Data Availability Not applicable.

\section{Compliance with Ethical Standards}

Conflict of Interest The authors declare that they have no conflict of interest.

Code Availability We have developed our code using the modeling and simulation framework of NetLogo, https://ccl.northwestern.edu/netlogo/. 


\section{References}

Acar, W., \& al-Gharaibeh, R. (2019). Internal and consulting information flows in the process of knowledge accumulation. International Journal of Knowledge Management, 15(1), 19-36.

Aggestam, L. (2006). Learning organization or knowledge management - Which came first, the chicken or the egg. Information Technology and Control, 35(3A), 295-302.

Ahmed, A. M. (2007). Group identity, social distance and intergroup bias. Journal of Economic Psychology, 28(3), 324-337.

Alavi, M., \& Leidner, D. (2001). Knowledge management and knowledge management systems: Conceptual foundations and research issues. MIS Quarterly, 25(1), 107-136.

Anand, A., Centobelli, P., \& Cerchione, R. (2020). Why should I share knowledge with others? A reviewbased framework on events leading to knowledge hiding. Journal of Organizational Change Management, 33(2), 379-399.

Bhaskar, V., \& Obara, I. (2002). Belief-based equilibria: The repeated prisoner's dilemma with private monitoring. Journal of Economic Theory, 102(1), 40-69.

Bligh, C. (2017). Leadership and trust. In J. Marques \& S. Dhiman (Eds.), Leadership today (pp. 21-42). Cham: Springer, Springer texts in business and economics.

Bock, G., Zmud, R., Kim, Y., \& Lee, J. (2005). Behavioral intention formation in knowledge sharing: Examining the roles of extrinsic motivators, social-psychological forces, and organizational climate. MIS Quarterly, 29(1), 87-111.

Brewer, M. B. (1979). In-group bias in the minimal intergroup situation: A cognitive-motivational analysis. Psychological Bulletin, 86(2), 307-324.

Cabrera, A., \& Cabrera, E. F. (2002). Knowledge-sharing dilemmas. Organization Studies, 23(5), 687-710.

Camerer, C. F. (1991). Does strategy research need game theory? Strategic Management Journal, 12(special issue), 137-152.

Cameron, P. (2000). Managing the wealth. CMA Management, 74(9), 46-49.

Chen, C.-J., \& Hung, S.-W. (2010). To give or to receive? Factors influencing members' knowledge sharing and community promotion in professional virtual communities. Information \& Management, 47(4), 226236.

Chuanrong, W., \& Deming, Z. (2009). Optimal decision making in the cooperation of organizations of knowledge transfer in innovation networks. Journal of Hunan University (Natural Science), 36(5), 89-92.

Clark, K., Kay, S., \& Sefton, M. (2001). When are Nash equilibria self-enforcing? An experimental analysis. Interna-tional Journal of Game Theory, 29, 495-515.

Cohen, W. M., \& Levinthal, D. A. (1990). Absorptive capacity: A new perspective on learning and innovation. Administrative Science Quarterly, 35(1), 128-152.

Cooper, R. W. (1999). Coordination games: Complementarities and macroeconomics. Cambridge: Cambridge University Press.

Cooper, R. W., DeJong, D. V., Forsythe, R., \& Ross, T. W. (1994). Alternative institutions for resolving coordination problems: Experimental evidence on forward induction and preplay communication. In J. W. Friedman (Ed.), Problems of coordination in economic activity (pp. 129-146). Boston: Kluwer.

Dahou, K., Hacini, I., \& Burgoyne, J. (2019). Knowledge management as a critical success factor in developing international companies' organizational learning capability. Journal of Workplace Learning, $31(\mathrm{n} 1), 2-16$.

Davenport, T., \& Prusak, L. (1998). Working knowledge: How organizations manage what they know. Boston: Harvard Business School Press.

Dawes, R. M., Orbell, J., \& Van de Kragt, A. J. C. (1988). Not me or thee but we: The importance of group identity in eliciting cooperation in dilemma situations: Experimental manipulations. Acta Psychologica, $68,83-97$.

D'Eredita, M. A., \& Barreto, C. (2006). How does tacit knowledge proliferate? An episode-based perspective. Organization Studies, 27(12), 1821-1841.

Dixon, N. M. (2000). Common knowledge: How companies thrive by sharing what they know. Boston: Harvard Business School Press.

Donninger, C. (1986). Is it always efficient to be nice? In A. Dickman \& P. Mitter (Eds.), Paradoxical effects of social behavior (pp. 123-134). Heidelberg: Physica Verlag.

Dreyfus, H., \& Dreyfus, S. (1986). Mind over machine: The power of human intuition and experience in the era of the computer. New York: The Free Press.

Fehr, E., \& Gachter, S. (2000). Fairness and retaliation: The economics of reciprocity. Journal of Economic Perspectives, 14, 159-181. 
Gintis, H. (2009). The bounds of reason: Game theory and the unification of the behavioral sciences. Princeton: Princeton University Press.

Goh, S. (2003). Improving organizational learning capability: Lessons from two case studies. The Learning Organization, 10(4), 216-227.

Gumus, M. (2007). The effect of communication on knowledge sharing in organizations. Journal of Knowledge Management Practice, 8(2).

Guo, Z. \& Sheffield, J. (2006). Habermasian inquiring system: Toward a general framework for knowledge management research. Proceedings of the $39^{\text {th }}$ Annual Hawaii International Conference on System Sceinces, Kauia, 7, 162c.

Hashim, K. F., \& Tan, F. B. (2015). The mediating role of trust and commitment on members' continuous knowledge sharing intention: A commitment-trust theory perspective. International Journal of Information Management, 35(2), 145-151.

Hau, Y. S., Kim, B., Lee, H., \& Kim, Y. G. (2012). The effects of individual motivations and social capital on employees' tacit and explicit knowledge sharing intentions. International Journal of Information Management, 33(2), 356-366.

Hsu, I.-C. (2006). Enhancing employee tendencies to share knowledge: Case studies of nine companies in Taiwan. International Journal of Information Management, 26(4), 326-338.

Hung, S.-Y., Durcikova, A., Lai, H.-M., \& Lin, W.-M. (2011). The influence of intrinsic and extrinsic motivation on individuals' knowledge sharing behavior. International Journal of Human-Computer Studies, 69, 415-427.

Jansson, F. (2015). What games support the evolution of an ingroup bias? Journal of Theoretical Biology, 373, $100-110$.

Kankanhalli, A., Tan, B. C. Y., \& Wei, K. K. (2005). Contributing knowledge to electronic knowledge repositories: An empirical investigation. MIS Quarterly, 29, 113-143.

Karkoulian, S., Messarra, L. C., \& McCarthy, R. (2013). The intriguing art of knowledge management and its relation to learning organizations. Journal of Knowledge Management, 17(4), 511-526.

Kim, D. (1993). The link between individual and organizational learning. Sloan Management Review, 35(1), $37-50$.

Kiyonari, T., Tanida, S., \& Yamagishi, T. (2000). Social exchange and reciprocity: Confusion or a heuristic? Evolution and Human Behavior, 21(6), 411-427.

Kline, P., \& Saunders, B. (1993). Ten steps to a learning organization. Arlington: Great Ocean Publishers.

Kollock, P. (1998). Social dilemmas: The anatomy of cooperation. Annual Review of Sociology, 24, $183-214$.

Kramer, R. M., Brewer, M. B., \& Hanna, B. A. (1995). Collective trust and collective action: The decision to trust as a social decision. In R. M. Kramer \& T. R. Tyler (Eds.), Trust in Organizations (pp. 357-389). Thousand Oaks: SAGE Publications, Inc..

Labedz, C., Cavaleri, S., \& Berry, G. (2011). Interactive knowledge management: Putting pragmatic policy planning in place. Journal of Knowledge Management, 15(4), 551-567.

Lam, A., \& Lambermont-Ford, J. (2010). Knowledge sharing in organisational contexts: A motivation-based perspective. Journal of Knowledge Management, 14(1), 51-66.

Lee, P., Gillespie, N., Mann, L., \& Wearing, A. (2010). Leadership and trust: The effect on knowledge sharing and team performance. Management Learning, 41(4), 473-491.

Li, Y.-M., \& Jhang-Li, J.-H. (2010). Knowledge sharing in communities of practice: a game theoretic analysis. European Journal of Operational Research, 207(2), 1052-1064.

Liu, X., Fang, Z., Zhang, N., Liu, K., \& Zhao, J. (2019). An evolutionary game model and its numerical simulation for collaborative innovation of multiple agents in carbon fiber industry in China. Sustainable Computing: Informatics and Systems, 24.

Lynne, M. M. (2001). Toward a theory of knowledge reuse: Types of knowledge reuse situations and factors in reuse success. Journal of Management Information System, 18(1), 57-93.

Mailath, G. J., \& Morris, S. (2006). Coordination failure in repeated games with almost-public monitoring. Theoretical Economics, 1(3), 311-340.

Maynard-Smith, J. (1982). Evolution and the theory of games. New York: Cambridge University Press.

McGurk, J., \& Baron, A. (2012). Knowledge management - Time to focus on purpose and motivation. Strategic HR Review, 11(6), 316-321.

Mueller, R. M., Spiliopoulou, M., \& Lenz, H-J. (2005). The influence of incentives and culture on knowledge sharing, Proceedings of the 38th Hawaii International Conference on System Sciences-2005.

Myerson, R. B. (1997). Game theory: Analysis of conflict. Cambridge: Harvard University Press.

Nonaka, I., \& Takeuchi, H. (1995). The knowledge-creating company: How Japanese companies create the dynamics of innovation. New York: Oxford University Press. 
Ogunmokun, O. A., Eluwole, K. K., Avci, T., Lasisi, T. T., \& Ikhide, J. E. (2020). Propensity to trust and knowledge sharing behavior: An evaluation of importance-performance analysis among Nigerian restaurant employees. Tourism Management Perspectives, 33, 100590.

Piccione, M. (2002). The repeated prisoner's dilemma with imperfect private monitoring. Journal of Economic Theory, 102(1), 70-83.

Politis, J. (2003). The connection between trust and knowledge management: What are its implications for team performance? Journal of Knowledge Management, 7(5), 55-66.

Rapoport, A., \& Chammah, A. M. (1965). Prisoner's dilemma: a study in conflict and cooperation. Ann Arbor: University of Michigan Press.

Rubinstein, R. (1991). Comments on the interpretation of game theory. Econometrica, 59(4), 909-924.

Sandhu, M. S., \& Ching, P. W. (2014). Relationship between individual cultural values and knowledge sharing in selected multinational companies in Malaysia. International Journal of Business and Economics, 13(1), 1-24.

Sen, A. K. (1967). Isolation, assurance and the social rate of discount. The Quarterly Journal of Economics, 81(1), 112-124.

Senge, P. (1994). The fifth discipline fieldbook: Strategies and tools for building a learning organization. New York: Doubleday.

Sharma, R. S., \& Bhattacharya, S. (2013). Knowledge dilemmas within organizations: Resolutions from game theory. Knowledge-Based Systems, 45, 100-113.

Skyrms, B. (2003). The stag hunt and the evolution of social structure. Cambridge: Cambridge University Press.

Suzuki, K., \& Harnisch, D. (1995). Measuring cognitive complexity: an analysis of performance-based assessment in mathematics. Paper presented at the 1995 Annual meeting of the American Educational Research Association (pp. 18-22). San Francisco.

Swan, J., Scarborough, H. \& Preston, J. (1999). Knowledge management - Next fad to forget people. Proceedings of the 7th European Conference on Information Systems, Copenhagen.

Tsoukas, H. (1996). The firm as a distributed knowledge system: A constructionist approach. Strategic Management Journal, 17(S2), 11-25.

Von Neumann, J., \& Morgenstern, O. (1944). The theory of games and economic behavior. Princeton: Princeton University Press.

Wang, C., Zuo, M. \& Quan, X. (2014). Knowledge sharing of senior employees in the context of task-based cooperation: A game theoretic analysis. Proceedings of the $18^{\text {th }}$ Pacific Asia Conference on Information Systems, Chengdu, paper 169.

Wasko, M. M., \& Faraj, S. (2000). It is what one does: Why people participate and help others in electronic communities of practice. Journal of Strategic Information Systems, 9, 155-173.

Watkins, K. E., \& Golembiewski, R. T. (1995). Rethinking organization development for the learning organization. The International Journal of Organizational Analysis, 3(1), 86-101.

Wolfe, C., \& Loraas, T. (2008). Knowledge sharing: The effects of incentives, environment, and person. Journal of Information Systems, 22(2), 53-76.

Wu, J., \& Xuan, H. (2004). A game-theoretic analysis of knowledge barter between staffs within firm. Studies in Science of Science, 12(22), 102-122.

Zhang, Z. (2018). Organizational culture and knowledge sharing: Design of incentives and business processes. Business Process Management Journal, 24(2), 384-399.

Zhang, M. J., \& Chen, H. (2018). To ask or not to ask: The roles of interpersonal trust in knowledge seeking. International Journal of Knowledge Management, 14(1), 1-16.

Zhang, X., Chen, Z., Vogel, D., Yuan, M., \& Guo, C. (2010). Knowledge-sharing reward dynamics in knowledge management systems: Game theory-based empirical validation. Human Factors and Ergonomics in Manufacturing \& Service Industries, 20(2), 103-122.

Zhang, X., De Pablos, P. O., \& Xu, Q. (2014). Culture effects on the knowledge sharing in multi-national virtual classes: A mixed method. Computers in Human Behavior, 31, 491-498.

Publisher's Note Springer Nature remains neutral with regard to jurisdictional claims in published maps and institutional affiliations. 


\section{Affiliations}

\section{Rami S. Al-Gharaibeh ${ }^{1}$ - Mostafa Z. Ali ${ }^{1}$}

Mostafa Z. Ali

mzali@just.edu.jo

1 Department of Computer Information Systems, Jordan University of Science and Technology, Irbid, Jordan 